\title{
Markov-Switching Three-Pass Regression Filter
}

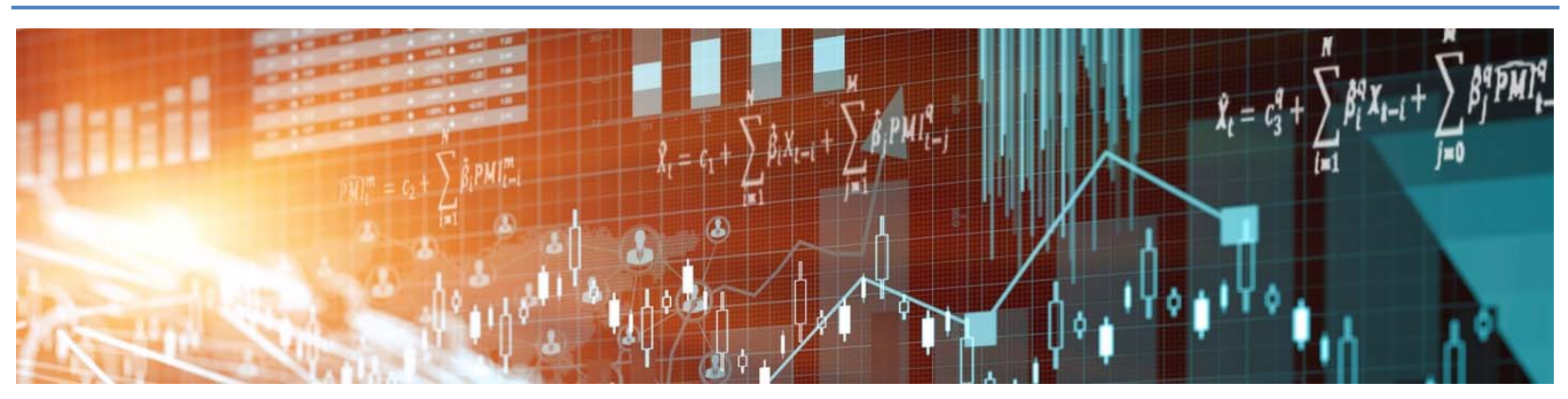

by Pierre Guérin, Danilo Leiva-Leon and Massimiliano Marcellino 
Bank of Canada Staff Working Paper 2017-13

April 2017

\title{
Markov-Switching Three-Pass Regression Filter
}

\author{
by
}

Pierre Guérin, ${ }^{1}$ Danilo Leiva-Leon ${ }^{2}$ and Massimiliano Marcellino ${ }^{3}$

1International Economic Analysis Department

Bank of Canada

Ottawa, Ontario, Canada K1A 0G9

pguerin@bankofcanada.ca

${ }^{2}$ Central Bank of Chile

dleiva@bcentral.cl

${ }^{3}$ Bocconi University

IGIER

and

CEPR

massimiliano.marcellino@unibocconi.it 


\section{Acknowledgements}

We would like to thank Dalibor Stevanovic, James MacKinnon, seminar participants at Queen's University and the 2016 conference of the Canadian Econometric Study Group held at Western University for helpful comments on a previous version of this paper. We also thank Agustín Díaz for excellent research assistance. The views expressed in this paper are those of the authors. No responsibility for them should be attributed to the Bank of Canada or the Central Bank of Chile. 


\begin{abstract}
We introduce a new approach for the estimation of high-dimensional factor models with regime-switching factor loadings by extending the linear three-pass regression filter to settings where parameters can vary according to Markov processes. The new method, denoted as Markov-switching three-pass regression filter (MS-3PRF), is suitable for datasets with large cross-sectional dimensions, since estimation and inference are straightforward, as opposed to existing regime-switching factor models where computational complexity limits applicability to few variables. In a Monte Carlo experiment, we study the finite sample properties of the MS-3PRF and find that it performs favourably compared with alternative modelling approaches whenever there is structural instability in factor loadings. For empirical applications, we consider forecasting economic activity and bilateral exchange rates, finding that the MS-3PRF approach is competitive in both cases.
\end{abstract}

\title{
Bank topic: Econometric and statistical methods JEL codes: C22; C23; C53
}

\section{Résumé}

Nous introduisons une nouvelle méthode d'estimation des modèles factoriels de large dimension avec changements de régimes en élargissant le filtre de régression linéaire à trois passages afin d'intégrer des paramètres qui peuvent varier en fonction de processus de Markov. Cette nouvelle méthode, appelée filtre de régression à trois passages avec changements de régimes markoviens (MS-3PRF), convient à des ensembles de données dotés d'importantes dimensions transversales car l'estimation et l'inférence s'y font directement. À l'inverse, les modèles factoriels à changements de régimes existants ne peuvent s'employer qu'avec un petit nombre de variables compte tenu de la complexité des algorithmes utilisés pour estimer ces modèles. Nous nous servons d'une expérience de Monte Carlo pour étudier les propriétés du filtre MS-3PRF sur un échantillon fini. Ce filtre donne de bons résultats par rapport aux autres méthodes de modélisation lorsque les pondérations factorielles présentent une instabilité structurelle. Pour l'application empirique, nous examinons la qualité des prévisions de l'activité économique et des taux de change bilatéraux : dans les deux cas, le filtre MS-3PRF se compare favorablement aux autres approches.

Sujet : Méthodes économétriques et statistiques

Codes JEL : C22 ; C23 ; C53 


\section{Non-Technical Summary}

This paper introduces a new approach for the estimation of high-dimensional factor models with regime-switching factor loadings. The premise of factor models is that the common dynamics of a large number of variables can be summarized in a relatively small number of unobserved factors. The literature on factor models has mostly concentrated on situations where the comovement among variables is assumed to be constant over time. However, there is now a large body of literature that has challenged the assumption of constant parameters to model the macroeconomic environment. It is also often found important to model time variation for macroeconomic forecasting.

The first contribution of this paper is to provide a new framework for the estimation of high-dimensional factor models with regime-switching parameters under classical inference. This method is particularly suitable in a forecasting context. In this approach, factors are extracted by modelling the regime-switching correlation of a large number of predictors with the variable to forecast. As such, the estimation of the factors takes into account how informative the predictors are for the variable one is interested in forecasting. A key advantage of our method is that it is computationally straightforward to implement.

The second contribution of this paper is to provide evidence that this new approach performs well when forecasting major U.S. macroeconomic variables. We also obtain predictive gains when forecasting major currencies based on a panel of exchange rates. This provides additional evidence of gains one can draw from the use of factor analysis to forecast exchange rates as well as the importance of modelling non-linearities in this context. 


\section{Introduction}

This paper introduces a new approach for the estimation of high-dimensional factor models with regime-switching factor loadings. The literature on factor models has mostly concentrated on situations where the comovement among variables is assumed to be constant over time. However, there is now a large body of literature that has challenged the assumption of constant parameters to model the macroeconomic environment (see, e.g., Sims (1993) or Canova (1993)), as well as the relevance of modelling time variation for macroeconomic forecasting (see, e.g., D'Agostino et al. (2013) and Aastveit et al. (2016)). The importance of incorporating time instabilities in large-scale factor models has gained traction in the literature in recent years (see, e.g., Eickmeier et al. (2015) and Mikkelsen et al. (2015)), but the number of works on this front remains relatively small. Moreover, this literature has so far been restricted to the estimation of models with time-varying factor loadings where time-variation is modelled using random-walk or autoregressive behaviours, which typically restrict the dynamics of time-variation to gradual changes in the factor loadings that may not be appropriate to all situations. The literature has also considered the estimation of factor models with temporal instability (structural breaks) in both factor loadings and the number of factors (see, e.g., Cheng et al. (2016)). In contrast, in this paper we consider factor loadings that vary according to regime-switching processes so as to model recurrent abrupt changes in factor loadings that are potentially highly relevant features in macroeconomic and financial variables.

Our modelling approach builds on Kelly and Pruitt (2015), who developed a new estimator for factor models - the three-pass regression filter (3PRF) - that relies on a series of ordinary least squares (OLS) regressions. As emphasized in Kelly and Pruitt (2015), the key difference between principal component analysis (PCA) and the 3PRF approach is that PCA summarizes the cross-sectional information based on the covariance within the predictors, whereas $3 \mathrm{PRF}$ condenses cross-sectional information based on the correlation of the predictors with the target variable of the forecasting exercise, thereby extending partial least squares. In this paper, we extend the 3PRF approach by introducing regime-switching parameters in the linear $3 \mathrm{PRF}$ filter. This new framework is denoted as Markov-switching three-pass regression filter (MS-3PRF). A key advantage of this approach is that it is well suited to handle high-dimensional factor models, as opposed to the existing regime-switching factor models that can handle only models with limited dimensions due to computational complexity (see, e.g., Camacho et al. (2012)). ${ }^{1}$ Our approach

\footnotetext{
${ }^{1}$ Groen and Kapetanios (2016) show that partial least squares (and Bayesian methods) perform better than principal components when forecasting based on a large data set with a weak factor structure. As partial least squares is obtained as a special case of the 3PRF (see Kelly and Pruitt (2015) for details), our method can also be adopted to introduce Markov switching in partial least squares regressions.
} 
is attractive in that our estimation strategy only requires estimating a series of univariate Markov-switching regressions. As such, it is computationally straightforward to implement and offers a great deal of flexibility in modelling time variation since we do not restrict the regime changes in the cross-sectional dimension to be governed by a single or a limited number of Markov chains. ${ }^{2}$

Empirically, we use the MS-3PRF approach for forecasting selected variables based on a large set of predictors. Since the seminal work of Stock and Watson (2002b), a large literature has developed to improve on the forecasting performance of the principal-component approach for macroeconomic forecasting (see, e.g., Forni et al. (2005) and De Mol et al. (2008), among many others). In a paper related to our work, Bai and $\mathrm{Ng}$ (2008) find improvements to the principal-component approach by using fewer but informative predictors. They also suggest that additional forecasting gains can be obtained when modelling non-linearities. The MS-3PRF approach is related to this strand of the literature given that factors are extracted by modelling the correlation of the predictors with the forecast target so that the estimation of the factors takes into account how informative the predictors are for the target variable. Moreover, the MS-3PRF approach captures non-linearities by modelling parameters that vary according to unobservable Markov chains.

This paper contributes to the literature along two main dimensions. First, we provide a new theoretical framework for the estimation of high-dimensional factor models with regime-switching parameters under classical inference. In a simulation experiment, we study the finite sample accuracy of the MS-3PRF forecasts compared with a number of alternatives. We find that the MS-3PRF performs well when there are instabilities in the data-generating process (DGP) modelled via regime-switching parameters. Second, we provide empirical evidence that the MS-3PRF performs well when forecasting major U.S. macroeconomic variables based on the McCracken and Ng (2015) data set. Moreover, when forecasting major currencies based on a panel of exchange rates, we also find predictive gains when using the MS-3PRF approach. This provides additional evidence of gains one can draw from the use of factor analysis to forecast exchange rates (see, e.g., Engel et al. (2015)) as well as the importance of modelling non-linearities in this context.

This paper is organized as follows: Section 2 introduces the MS-3PRF approach and discusses its main features. Section 3 presents a Monte Carlo experiment to study the finite sample accuracy of the MS-3PRF. Section 4 gathers empirical applications devoted to macroeconomic and exchange rate forecasting. Section 5 concludes.

\footnotetext{
${ }^{2}$ For example, extracting one factor from the MS-3PRF approach using a panel of 130 macroeconomic and financial variables with gross domestic product (GDP) growth as a target proxy takes about 350 seconds using a laptop with a $2.7 \mathrm{GHz}$ processor and $16 \mathrm{~GB}$ RAM.
} 


\section{Markov-Switching Three-Pass Regression Filter}

\subsection{The algorithm}

There is now a growing literature on dynamic factor models with time-varying parameters. For example, in a Bayesian setting, Del Negro and Otrok (2008) first introduced a dynamic factor model with time-varying factor loadings. In a classical context, see Mikkelsen et al. (2015) and Eickmeier et al. (2015). However, the literature on regimeswitching dynamic factor models is limited and, more importantly, restricted to small-scale models (see, e.g., Chauvet (1998), Camacho et al. (2012) or Barnett et al. (2016), who use fewer than 10 variables and focus only on switches in the parameters governing the factor dynamics and not the factor loadings). ${ }^{3}$ The same is true for vector autoregression (VAR) models: while there is now a large (both methodological and empirical) literature on time-varying parameter VAR models, the literature using regime-switching VAR models is a lot narrower, although there are a few noticeable exceptions (see, e.g., Sims and Zha (2006) and Hubrich and Tetlow (2015)).

One key reason for the absence of a significant literature on large-scale Markov-switching factor models relates to the computational challenges associated with the estimation of such models. We present here the Markov-switching three-pass regression filter, which circumvents these difficulties and offers a flexible approach in that we impose very few restrictions on the Markov processes driving the changes in the parameters of the model.

The type of setting we have in mind can be described informally as follows: There is a relatively large number $N$ of predictors $\mathbf{x}$ from which we want to extract factors so as to forecast a target variable $y$. While $\mathbf{x}$ depends on two sets of common factors, say $f$ and $g$ (plus idiosyncratic components), $y$ depends only on $f$, so we would like to extract only $f$ from $\mathbf{x}$. In addition, there exist proxi variables, $\mathbf{z}$, whose common components are also driven only by $f$. This setting is the same as that in Kelly and Pruitt (2015), who introduced the linear 3PRF for estimation of $f$ and forecasting of $y$, but the key novelty is that we include time variation in the model parameters via Markov processes.

\footnotetext{
${ }^{3}$ In a Bayesian context, Guérin and Leiva-Leon (2016) develop an algorithm to estimate a highdimensional factor-augmented VAR model with regime-switching parameters in the factor loadings to study the interactions between monetary policy, the stock market and the connectedness of industry-level stock returns. See also Von Ganske (2016), who introduces regime-switching parameters in partial least squares regressions from a Bayesian perspective so as to forecast industry stock returns. Using a Bayesian framework, Hamilton and Owyang (2012) develop a framework for modelling common Markov-switching components in panel data sets with large cross-sectional and time-series dimensions to estimate turning points in U.S. state-level employment data.
} 
More formally, let us consider the following model:

$$
\begin{aligned}
y_{t+1} & =\beta_{0}\left(S_{t}\right)+\boldsymbol{\beta}^{\prime}\left(S_{t}\right) \mathbf{F}_{t}+\eta_{t+1}\left(S_{t}\right), \\
\mathbf{z}_{t} & =\lambda_{0}\left(\mathbf{S}_{t}\right)+\boldsymbol{\Lambda}\left(\mathbf{S}_{\mathbf{t}}\right) \mathbf{F}_{t}+\omega_{t}\left(\mathbf{S}_{t}\right), \\
\mathbf{x}_{t} & =\phi_{0}\left(\mathbf{S}_{t}\right)+\boldsymbol{\Phi}\left(\mathbf{S}_{\mathbf{t}}\right) \mathbf{F}_{t}+\varepsilon_{t}\left(\mathbf{S}_{t}\right),
\end{aligned}
$$

where $y$ is the target variable of interest; $\mathbf{F}_{t}=\left(\mathbf{f}_{t}^{\prime}, \mathbf{g}_{t}^{\prime}\right)^{\prime}$ are the $K=K_{f}+K_{g}$ common driving forces of all variables, the unobservable factors; $S_{t}$ denotes a standard Markov chain driving the parameters of the forecasting equation, while $\mathbf{S}_{t}=\left(S_{1, t}, S_{2, t}, \ldots, S_{N, t}\right)^{\prime}$ is a vector containing variable-specific Markov chains with $M$ regimes driving the parameters of the factor equations; each Markov chain is governed by its own $M \times M$ transition probability matrix,

$$
P_{i}=\left(\begin{array}{cccc}
p_{i, 11} & p_{i, 21} & \cdots & p_{i, M 1} \\
p_{i, 12} & p_{i, 22} & \cdots & p_{i, M 2} \\
\vdots & \vdots & \ddots & \vdots \\
p_{i, 1 M} & p_{i, 2 M} & \cdots & p_{i, M M}
\end{array}\right),
$$

for $i=1,2, \ldots, N ; \boldsymbol{\beta}\left(S_{t}\right)=\left(\boldsymbol{\beta}_{f}^{\prime}\left(S_{t}\right), \mathbf{0}^{\prime}\right)^{\prime}$, so that $y$ depends only on $\mathbf{f} ; \mathbf{z}$ is a small set of $L$ proxies that are driven by the same underlying forces as $y$, so that $\boldsymbol{\Lambda}\left(\mathbf{S}_{\mathbf{t}}\right)=\left(\boldsymbol{\Lambda}_{f}\left(\mathbf{S}_{t}\right), \mathbf{0}\right) ; \mathbf{x}_{t}$ is a large set of $N$ variables, driven by both $\mathbf{f}_{t}$ and $\mathbf{g}_{t}$; and $t=1, \ldots, T$.

To achieve identification, when $N$ and $T$ diverge, the covariance of the loadings is assumed to be the identity matrix in each state, and the factors are orthogonal to one another. ${ }^{4}$ For the sake of space, we refer to Kelly and Pruitt (2015) for precise conditions on the factors, allowed temporal and cross-sectional dependence of the residuals, and the existence of proper central limit theorems.

Given the model in equations 1 to 3, our algorithm for the MS-3PRF model consists of the following three steps:

- Step 1: Time-series regressions of each element of $\mathbf{x}, x_{i}$, on $\mathbf{z}$; that is, run $N$ Markovswitching regressions

$$
x_{i, t}=\phi_{0, i}\left(S_{i, t}\right)+\mathbf{z}_{t}^{\prime} \phi_{i}\left(S_{i, t}\right)+\epsilon_{i, t}\left(S_{i, t}\right)
$$

\footnotetext{
${ }^{4}$ More precisely, defining $\mathbf{J}_{T}=\mathbf{I}_{T}-\frac{1}{T} \iota_{T} \iota_{T}^{\prime}$, where $\mathbf{I}_{T}$ is a $T$-dimensional identity matrix and $\iota_{T}$ is a $T$-vector of ones (and similarly $\left.\mathbf{J}_{N}\right)$, and assuming that $N^{-1} \boldsymbol{\Phi}^{\prime}\left(S_{t}\right) \mathbf{J}_{N} \boldsymbol{\Phi}\left(S_{t}\right) \underset{N \longrightarrow \infty}{\longrightarrow} \mathbf{P}\left(S_{t}\right)$, $N^{-1} \boldsymbol{\Phi}^{\prime}\left(S_{t}\right) \mathbf{J}_{N} \phi_{0}\left(S_{t}\right) \underset{N \longrightarrow \infty}{\longrightarrow} \mathbf{P}_{1}\left(S_{t}\right), T^{-1} \mathbf{F}^{\prime} \mathbf{J}_{T} \mathbf{F} \underset{T \longrightarrow \infty}{\longrightarrow} \Delta_{F}$, for identification we require, as did Kelly and Pruitt (2015), that $\mathbf{P}\left(\mathbf{S}_{\mathbf{t}}\right)=\mathbf{I}, \mathbf{P}_{1}\left(S_{t}\right)=\mathbf{0}$, and $\Delta_{F}$ is diagonal and positive definite, and each diagonal element is unique.
} 
where $i=\{1, \ldots, N\}, \epsilon_{i, t} \mid S_{i, t} \sim N I D\left(0, \sigma^{2}\left(S_{i, t}\right)\right)$, and keep the maximum likelihood estimate of $\phi_{i}\left(S_{i, t}\right)$, denoted by $\widehat{\phi}_{i}\left(S_{i, t}\right)$. All regime-switching models are estimated via (pseudo) maximum likelihood, and we make a normality assumption about the disturbances to write down the log-likelihood function, which is not required when estimating the linear version of the $3 \mathrm{PRF}$. As mentioned previously, $S_{i, t}$ is a standard Markov chain with $M$ regimes and dynamics driven by constant transition probabilities. It is important to stress that the estimated latent processes $S_{i, t}$ differ across all cross-section units $i$. The pattern of the regime changes in the factor loadings is therefore left unrestricted as opposed to assuming that the changes in the parameters $\phi_{0, i}$ and $\phi_{i}$ are governed by a single (or a limited number of) Markov chain(s) across all cross-section units. Moreover, a different number of regimes could be used across the $N$ first-pass regressions. As such, the MS-3PRF approach offers a great deal of flexibility in modelling regime changes.

- Step 2: Cross-section regressions of $x_{i, t}$ on $\hat{\phi}_{i, t}$; that is, run $T$ linear regressions

$$
x_{i, t}=\alpha_{0, t}+\hat{\phi}_{i, t}^{\prime} \mathbf{F}_{t}+\epsilon_{i, t}
$$

where $t=\{1, \ldots, T\}$, and keep (for each $t$ ) the OLS estimates $\mathbf{F}_{t}$. In this step, the time-varying factor loadings $\hat{\phi}_{i, t}$ can be obtained from the first step of the algorithm by following two alternatives. First, as a weighted average of the regime-specific factor loadings:

$$
\hat{\boldsymbol{\phi}}_{i, t}=\sum_{j=1}^{M} \hat{\boldsymbol{\phi}}_{i}\left(S_{i, t}=j\right) P\left(S_{i, t}=j \mid \Omega_{T}\right),
$$

where $P\left(S_{i, t}=j \mid \Omega_{T}\right)$ is the smoothed probability of being in regime $j$ given the full sample information $\Omega_{T}$. Second, as a selected loading:

$$
\hat{\boldsymbol{\phi}}_{i, t}=\sum_{j=1}^{M} \hat{\boldsymbol{\phi}}_{i}\left(S_{i, t}=j\right) I\left(P\left(S_{i, t}=j \mid \Omega_{T}\right)\right),
$$

where $I(\cdot)$ is an indicator function that selects the regime with the highest smoothed probability, $P\left(S_{i, t}=j \mid \Omega_{T}\right)$, at time $t$.

- Step 3: Time-series regressions of $y_{t}$ on $\hat{\mathbf{F}}_{t-h}$; that is, run one Markov-switching regression for each forecast horizon of interest, $h$ :

$$
y_{t}=\beta_{0}\left(S_{t}\right)+\hat{\mathbf{F}}_{t-h}^{\prime} \boldsymbol{\beta}\left(S_{t}\right)+\eta_{t}\left(S_{t}\right)
$$

keep the maximum likelihood estimates $\beta_{0}\left(S_{t}\right)$ and $\boldsymbol{\beta}\left(S_{t}\right)$, and calculate the forecast 
$\hat{y}_{T+h \mid T}$ as:

$$
\hat{y}_{T+h \mid T}=\sum_{j=1}^{M}\left(P\left(S_{T+h}=j \mid \Omega_{T}\right) \hat{\beta}_{0}\left(S_{T+h}=j\right)+P\left(S_{T+h}=j \mid \Omega_{T}\right) \hat{\mathbf{F}}_{t}^{\prime} \hat{\boldsymbol{\beta}}\left(S_{T+h}=j\right)\right),
$$

where $P\left(S_{T+h}=j \mid \Omega_{T}\right)$ is the predicted probability of being in regime $j h$-step-ahead given the information available up to time $T, \Omega_{T}$.

In the third pass of the algorithm, the Markov chain $S_{t}$ allows us to model time variation in the intercept of the forecasting regression, which is a common source of forecast failure. Changes in the slope parameters $\boldsymbol{\beta}$ are relevant in that this also allows us to model time

variation in the predictive power of the estimated factors $\hat{\mathbf{F}}_{t}$ for the target variable $y_{t+1}$. Note that one can estimate a linear model in the third step. We denote this approach as "MS-3PRF (first pass)," while "MS-3PRF (first and third passes)" refers to considering regime changes in both the first and the third pass.

\subsection{Assumptions and theoretical properties}

The algorithm outlined in the previous subsection rests on a number of assumptions, including the choice of the number of factors and proxi variables to be used in the first step of the algorithm, as well as the number of regimes to consider for the MS-3PRF.

There are several ways to assess the number of regimes in a Markov-switching regression under a classical framework. Just to mention a few, Cho and White (2007) and Carter and Steigewald (2012) suggest the use of a quasi-likelihood ratio test; however, they ignore the Markov property of the variable $S_{t}$. Other alternatives consist in calculating goodnessof-fit measures that trade off the fit of the likelihood against the number of parameters (e.g., Smith et al. (2006)). For ease of illustration of the proposed approach, throughout the simulation exercises and empirical applications, we leave aside this complication and assume that predictor variables experience either $M=1$ or $M=2$ regimes. However, the framework can be generalized accordingly.

For the choice of the proxi variables, when there is just one $\mathbf{f}_{t}$ factor, Kelly and Pruitt (2015) suggest using directly the target variable $y$ as proxy z. From a predictive point of view, this is a natural choice, since, in this context, one wants to extract a factor that summarizes how related the predictors are to the the predicted variable. They refer to this case as target-proxy $3 \mathrm{PRF}$. In the case of more factors, they propose using either economic theory to select indicators correlated with the target variable $y$, or an automated procedure that can be implemented with the following steps, indicating a proxy by $z_{j}$ with $j=1, \ldots, L$. 
- Pass 1: Set $z_{1}=y$; get the $3 \mathrm{PRF}$ forecast $\widehat{y}_{t}^{1}$, and the associated residuals $e_{t}^{1}=y_{t}-\widehat{y}_{t}^{1}$.

- Pass 2: Set $z_{2}=e^{1}$; get the $3 \mathrm{PRF}$ forecast $\widehat{y}_{t}^{2}$ using $z_{1}$ and $z_{2}$ as proxies. Get the associated residuals $e_{t}^{2}=y_{t}-\widehat{y}_{t}^{2}$.

- ...

- Pass L: Set $z_{L}=e^{L-1}$; get the $3 \mathrm{PRF}$ forecast $\widehat{y}_{t}^{L}$ using $z_{1}, z_{2}, \ldots z_{L}$ as proxies.

For the choice of the number of factors, Kelly and Pruitt (2015) use appropriate information criteria with asymptotic optimality properties. However, empirically it can be more informative to assess the performance of different numbers of factors. As in the case of PCA, using more factors than needed reduces forecast efficiency in finite samples but does not introduce a bias, while using fewer factors generates an omitted-variable problem and therefore biases both the estimators of the loadings and the forecasts.

Kelly and Pruitt (2015) develop asymptotic theory for the linear 3PRF approach, showing that the $3 \mathrm{PRF}$-based forecast converges in probability to the infeasible best forecast as cross-section $N$ and sample size $T$ become large. However, we need additional special conditions to be able to claim that their consistency results could be extended to the non-linear case. Specifically, we need consistency of the parameter estimators for the Markov-switching models in steps 1 and 3. Douc et al. (2004) establish results concerning the consistency and asymptotic normality of the maximum likelihood estimator in Markovswitching models. For the general case of hidden Markov models, Leroux (1992) proved the consistency of the maximum likelihood estimator under mild regularity conditions. For ease of notation, we suppress the $i$ subscript and assume that the same conditions apply to all the predictor variables in $\mathbf{x}$. In particular, for any given variable $x_{t}$, let $\{f(\cdot, \theta): \theta \in \Theta\}$ be a family of densities on a Euclidean space with respect to $\theta_{1}, \theta_{2}, \ldots, \theta_{M}$ elements of $\Theta$.

The characteristics of the model are parameterized by $\phi$, which belongs to a parameter space $\Phi$; that is, we have $p_{j k}(\phi)$, for $j, k=1,2, \ldots, M$, and $\theta_{j}(\phi) \in \Theta$, for $j=1,2, \ldots, M$. The usual case is $\phi=\left(p_{11}, p_{12}, \ldots, p_{M M}, \theta_{1}, \theta_{2}, \ldots, \theta_{M}\right)$, and $p_{j k}(\cdot)$ and $\theta_{j}(\cdot)$ equal to coordinate projections. Letting the true parameter value be denoted by $\phi^{*}$, we assume the following conditions:

- Assumption 1: The $M \times M$ transition probability matrix, $\left[p_{j k}\left(\phi^{*}\right)\right]$, is irreducible.

- Assumption 2: The family of mixtures of at most $M$ elements of $\{f(y, \theta): \theta \in$ $\Theta\}$ is identifiable. In other words, the finite mixture with $M$ or fewer components determines a unique distribution. 
- Assumption 3: For each $x_{t}$, the density $f\left(x_{t}, \cdot\right)$ is continuous and vanishes at infinity.

- Assumption 4: For each $j, k, p_{j k}(\cdot)$ and $\theta_{j}(\cdot)$ are continuous.

- Assumption 5: $E_{\phi^{*}}\left[\left|\log \left(f\left(X_{1}, \theta_{j}\left(\phi^{*}\right)\right)\right)\right|\right]<\infty$ for $j=1,2, . ., M$.

- Assumption 6: For every $\theta \in \Theta, E_{\phi^{*}}\left[\sup _{\left\|\theta-\theta^{\prime}\right\|<\delta}\left(\log \left(f\left(X_{1}, \theta^{\prime}\right)\right)\right)^{+}\right]<\infty$, for some $\delta>0 .^{5}$

Leroux (1992) proved that under assumptions 1 to 6 , the maximum likelihood estimator, $\hat{\phi}$, converges to the true parameter value, $\phi^{*}$, with probability one.

Hence, based on this consistency result of the Markov-switching parameters in the first and third steps, under the above assumptions 1 to 6 and the assumptions in Kelly and Pruitt (2015), the asymptotic results from Kelly and Pruitt (2015) follow through, and thereby the MS-3PRF conserves the theoretical properties from the linear 3PRF. However, since in practical applications both $T$ and $N$ are finite, it is important to assess also the finite sample performance of the MS-3PRF, which is what we do in the next section.

\section{Monte Carlo Simulations}

In this section, we conduct Monte Carlo simulations to evaluate the finite sample properties of the MS-3PRF, focusing on its predictive performance. We compare the MS-3PRF with competing approaches that have proved to be successful in dealing with large data sets, such as the linear 3PRF and PCA. We also use two additional benchmark models - targeted PCA (TPCA) and a least angle regression approach, PC-LARS - from which factors are extracted by the method of principal component from a smaller set of predictors than the $N$ predictors used by PCA. These two additional methods are described in the appendix; Appendix A.1 describes the hard-thresholding approach (TPCA), and Appendix A.2 outlines the soft-thresholding approach (PC-LARS). Our simulation exercises compute the full-sample mean square forecasting errors (MSFE) to predict the target variable, $y_{t}$, that is generated based on a large set of predictors, $\mathbf{x}_{t}=\left(x_{1, t}, x_{2, t}, \ldots, x_{N, t}\right)^{\prime}$, driven by a factor structure with regime-switching in the loadings.

\footnotetext{
${ }^{5}$ Where $\|\cdot\|$ is the Euclidean distance, and $w^{+}=\max \{w, 0\}$.
} 


\subsection{Design}

The data on $\mathbf{x}_{t}$ and $y_{t}$, for $t=\{1,2, \ldots, T\}$, are generated following the factor structure proposed in Bates et al. (2013) and Kelly and Pruitt (2015):

$$
\begin{aligned}
\mathbf{x}_{t} & =\Phi_{t} F_{t}+\varepsilon_{t}, \\
y_{t+1} & =\Lambda F_{t}+\eta_{t}
\end{aligned}
$$

where $F_{t}=\left(f_{t}, \mathbf{g}_{t}^{\prime}\right)^{\prime}, \Phi_{t}=\left(\Phi_{f, t}, \Phi_{g, t}\right)$ and $\Lambda=(1, \mathbf{0})$. The relevant and irrelevant factors are generated according to the following dynamics, respectively:

$$
\begin{aligned}
& f_{t}=\rho_{f} f_{t-1}+u_{f, t}, \\
& \mathbf{g}_{t}=\rho_{g} \mathbf{g}_{t-1}+\mathbf{u}_{g, t},
\end{aligned}
$$

where $u_{f, t} \sim N(0,1)$, and $\mathbf{u}_{g, t} \sim N\left(0, \boldsymbol{\Sigma}_{g}\right)$, with $u_{f, t}$ and $\mathbf{u}_{g, t}$ uncorrelated. We consider $K_{g}=4$ irrelevant factors and $K_{f}=1$ relevant factor. The parameters in $\Sigma_{g}$ are chosen so that irrelevant factors are dominant; that is, their variances are 1.25, 1.75, 2.25 and 2.75 times larger than the relevant factor. The idiosyncratic terms are assumed to follow autoregressive dynamics,

$$
\varepsilon_{i t}=\alpha \varepsilon_{i, t-1}+v_{i, t}
$$

and to be cross-sectionally correlated; that is, $v_{t}=\left(v_{1, t}, v_{1, t}, \ldots, v_{N, t}\right)^{\prime}$, and it is independent and identically distributed (i.i.d.) normally distributed with covariance matrix $\Omega=\left(\beta^{|i-j|}\right)_{i j}$, as in Amengual and Watson (2007). The starting values for the factors and idiosyncratic terms $f_{0}, \mathbf{g}_{0}, \varepsilon_{i 0}$ are drawn from their respective stationary distributions. The disturbances, $\eta_{t}$, associated to the target variable equation are i.i.d. normally distributed with a variance, $\sigma_{\eta}^{2}$, that is adjusted to ensure that the infeasible best forecast has a $R^{2}$ of 50 per cent. The free parameters of our Monte Carlo simulations are $\rho_{f}, \rho_{g}, \alpha, \beta, N$ and T. In line with Stock and Watson (2002a), Bates et al. (2013) and Kelly and Pruitt (2015), we consider $\rho_{f}=\{0.3,0.9\}, \rho_{g}=\{0.3,0.9\}, \alpha=\{0.3,0.9\}, \beta=\{0,0.5\}, N=\{100,200\}$ and $T=\{100,200\}$.

The factor loadings, collected in $\Phi_{t}$, experience changes between two regimes over time,

$$
\Phi_{t}=\Phi_{1} \mathbf{S}_{t}+\Phi_{2}\left(1-\mathbf{S}_{t}\right)
$$

where $\mathbf{S}_{t}=\left(S_{1, t}, S_{2, t}, \ldots, S_{N, t}\right)$ contains $N$ dichotomous state variables, each following distinct dynamics according to a first-order Markov chain. Since the data in $\mathbf{x}_{t}$ are generated from a factor structure, it is mechanically subject to a certain degree of comovement. Therefore, the non-linear relationship between the data, $\mathbf{x}_{t}$, and the factors, $F_{t}$, measured by the factor loadings, $\Phi_{t}$, may also experience a certain degree of comovement. 
To provide a more realistic DGP that is relevant for economic data where data are generally weakly dependent (as opposed to i.i.d.), we model comovement in the factor loadings, which is translated in modelling comovement in the Markovian state variables contained in $\mathbf{S}_{t}$. In doing so, we let $\tilde{S}_{i, t}$ be the state vector of the $i$-th sequence at time $t$. If the $i$-th sequence is in state 1 at time $t$, then we write $\tilde{S}_{i, t}=(1,0)^{\prime}$, and if it is in state 2 at time $t$, then we write $\tilde{S}_{i, t}=(0,1)^{\prime}$. First, we generate a "seed" sequence variable, $\tilde{S}_{0, t}$. For time $t$, we compute $(q, 1-q)^{\prime}=P_{00} \tilde{S}_{0, t}$, where

$$
P_{00}=\left(\begin{array}{cc}
p_{11} & 1-p_{22} \\
1-p_{11} & p_{22}
\end{array}\right)
$$

is the transition probability matrix, and the realization of the sequence at time $t+1$ is defined as

$$
\tilde{S}_{0, t+1}=\left\{\begin{array}{cc}
(1,0)^{\prime} & \text { If } q \geq \theta, \\
(0,1)^{\prime} \text { otherwise }
\end{array}\right.
$$

where $\theta$ is drawn from a $U[0,1]$. Next, we generate a Markov chain, $\tilde{S}_{i, t}$, conditional on the dynamics of $\tilde{S}_{0, t}$, using the following system:

$$
\left[\begin{array}{c}
\left(q_{0}, 1-q_{0}\right)^{\prime} \\
\left(q_{i}, 1-q_{i}\right)^{\prime}
\end{array}\right]=\left[\begin{array}{cc}
\lambda_{00} P_{00} & \lambda_{0 i} P_{0 i} \\
\lambda_{i 0} P_{i 0} & \lambda_{i i} P_{i i}
\end{array}\right]\left[\begin{array}{c}
\tilde{S}_{0, t} \\
\tilde{S}_{i, t}
\end{array}\right],
$$

where the coefficients $\lambda$ measure the comovement between both Markov chains, with $\lambda_{j k} \geq$ 0 , and $\sum_{k=1}^{2} \lambda_{j k}=1$. The matrix $P_{j k}$ collects the transition probabilities from the states in the $k$-th sequence to the states in the $j$-th sequence. ${ }^{6}$ Accordingly, $q_{k}$ represents the state probability distribution of the $k$-th sequence at time $t+1$, from which the realization $\tilde{S}_{i, t+1}$ can be generated as follows:

$$
\tilde{S}_{i, t+1}=\left\{\begin{array}{cc}
(1,0)^{\prime} & \text { If } q_{i} \geq \theta \\
(0,1)^{\prime} & \text { Otherwise }
\end{array} .\right.
$$

For simplicity, we assume that $P_{0 i}=P_{i 0}=P_{i i}=P_{00}$, and that $p_{11}=p_{22}$, with $p_{11}=0.9$. Also, we set $\lambda_{00}=\lambda_{i i}=0.2$ to induce a relatively large degree of comovement between the state variables. Given $\tilde{S}_{0, t}$, we repeat the same procedure for $i=\{1,2, \ldots, N\}$, to get all the elements in $\mathbf{S}_{t}$. Finally, the elements in $\Phi_{\kappa}$, for $\kappa=\{1,2\}$ are generated from a $N\left(\phi_{\kappa}, \sigma_{\phi}\right)$ with $\phi_{1}=0.5, \phi_{2}=1.5$, and $\sigma_{\phi}=0.1$.

We also study the case where $\mathbf{x}_{t}$ and $y_{t}$ are generated by following the same processes described above but with the factor loadings being driven by Markov chains that are totally independent of each other and that do not experience comovement. Finally, we assess the performance of the proposed non-linear approaches in estimating the factors under different features of regime-switching factor-loading dynamics.

\footnotetext{
${ }^{6}$ Ching et al. (2002) proposed a multivariate Markov chain approach for modelling multiple categorical data sequences.
} 


\subsection{Models and evaluation criteria}

We perform $L=500$ Monte Carlo replications for each configuration of parameters $\rho_{f}$, $\rho_{g}, \alpha$ and $\beta$ and sample sizes $T$ and $N$. Once $\mathbf{x}_{t}$ and $y_{t}$ are generated, we apply the MS$3 \mathrm{PRF}$ to extract the factor and predict the target variable. In particular, first, we estimate a time-series (Markov-switching) regression, $x_{i, t}=\mathbf{z}_{t}^{\prime} \boldsymbol{\phi}_{i}\left(S_{i, t}\right)+\epsilon_{i, t}$, for $i=\{1,2, \ldots, N\}$. For simplicity, we take the proxy variable as the target variable, $\mathbf{z}_{t}^{\prime}=y_{t}$. Second, we run a cross-section OLS regression, $x_{i, t}=\hat{\boldsymbol{\phi}}_{i, t}^{\prime} \mathbf{F}_{t}+\epsilon_{i, t}$, for $t=1,2, \ldots, T$, using the weighted average of the regime-switching factor loadings obtained in the previous step. Third, we run a time-series OLS regression, $y_{t}=\hat{\mathbf{F}}_{t-1}^{\prime} \boldsymbol{\beta}+\eta_{t}$, and produce the forecast $\hat{y}_{t+1}=\hat{\mathbf{F}}_{t}^{\prime} \hat{\boldsymbol{\beta}}$, obtained with the MS-3PRF approach introduced in this paper. Also, we produce forecasts with the version of the MS-3PRF when the loadings are selected instead of being averaged (MSS-3PRF); that is, the time-varying loadings are set to the regime-specific loadings of the most likely regime. To ease the computational burden, in our Monte Carlo simulations, we do not model regime switches in the third pass of the algorithm. This is not detrimental to our simulation exercise, since we are interested only in studying situations characterized by instabilities in the factor loadings (and not time instability in the relationship between the predicted variable and the predictors). However, in the empirical applications, we consider the case of regime switches in the third pass.

We compare the predictive performance of the two variants of our proposed method with several benchmark methodologies. First, we compute the forecast obtained with the linear version of the 3PRF proposed in Kelly and Pruitt (2015). Second, Bates et al. (2013) show that PCA methods can be applied to consistently estimate dynamic factor models under certain instabilities in the loadings. Therefore, we compute the forecast obtained with the method of principal components. Third, Bai and Ng (2008) argue that the principal components methodology, as it stands, does not take into account the predictive ability of $\mathbf{x}_{t}$ for $y_{t+h}$ when the factors are estimated. Therefore, Bai and Ng (2008) propose using only predictors that are informative for $y_{t}$ in estimating the factors in order to take explicitly into account that the object of interest is ultimately the forecast of $y_{t}$. Accordingly, we also compute the forecast obtained with TPCA. Fourth, we consider the elastic net softthresholding rules, which are special cases of the LARS algorithm developed in Efron et al. (2004), and compute the forecast using the PC-LARS method. Ultimately, our focus is on comparing the median in-sample MSFE over the $L$ replications associated with each of the six methods (two MS-3PRF approaches and four competitors) to evaluate their relative predictive performance. Finally, in our simulation experiments, we assume that the number of relevant factors and the number of regimes are known (i.e., across all procedures, we extract one factor, and the non-linear models consider the case of two regimes). 


\subsection{Results}

Table 1 reports the simulation results associated with the different configurations of parameters, methodologies and degrees of instability (regime switching) in the data. In particular, the upper part of Table 1 presents the MSFE for the cases when all factor loadings exhibit regime-switching dynamics. First, the MSS-3PRF exhibits the lowest MSFE for most of the cases, indicating that it performs best in terms of predictive performance. In particular, the MSS-3PRF outperforms the MS-3PRF, linear 3PRF, PCA, TPCA and PC-LARS. Notice also that, in general, the MS-3PRF exhibits the second-best forecasting performance, suggesting that the non-linear frameworks, MS-3PRF and especially MSS$3 \mathrm{PRF}$, are able to capture in a better way instabilities in the relationship between the set of predictors and its common factor.

Second, TPCA tends to provide the best performance when the irrelevant factors, and the idiosyncratic terms, have a low autocorrelation, but the relevant factor is highly autocorrelated. If the irrelevant factors behave like white-noise processes, while the relevant factor experiences a high autocorrelation, then TPCA would be able to easily identify the variables highly associated with the relevant factor so as to obtain a reliable estimation of the relevant factor, as long as the target variable to be predicted, $y_{t}$, does not act as a white noise. Also notice that in most cases the linear 3PRF outperforms PCA and PCLARS. This implies that in the presence of instabilities in the loadings, the linear 3PRF takes better advantage of both time-series and cross-sectional dimensions to provide a more accurate estimation of the underlying factor than PCA and PC-LARS.

Third, the scenarios associated with low autocorrelation in the irrelevant factors, $\rho_{g}$, yield the highest MSFE. The fact that irrelevant factors (i) behave close to a white noise, (ii) are linked to $\mathbf{x}_{t}$ through regime-switching loadings and (iii) are dominant makes them able to introduce a relatively large amount of noise into the set of predictors, creating more difficulty for all the methods to provide more accurate estimates of the underlying relevant factor and consequently better forecasts for $y_{t}$. In particular, when not only the irrelevant factors but also the idiosyncratic terms are closer to behaving as a white noise (that is, $\rho_{g}=0.3$ and $\alpha=0.3$ ), the forecasting performance of all methods deteriorates due to the reason just described. These results indicate that in the presence of instabilities in the loadings, a lower autocorrelation in any of the components driving the observed data (predictors and target variables) is detrimental to the predictive performance of all factor-extraction methods studied in this paper.

Fourth, the performance of the methods varies with the size of both cross-sectional and time-series dimensions. In particular, while a larger $N$ helps to improve the predictive ability of the methods, a larger $T$ appears to be detrimental to the predictive ability. 
Notice that when holding constant the cross-sectional dimension $N$ and letting grow the time-series dimension $T$-that is, when comparing the case of $T=100, N=100$ versus the case of $T=200, N=100$ - there is an overall increase in the corresponding MSFE. However, when keeping $T$ constant and letting $N$ grow - that is, when comparing the case of $T=100, N=100$ versus the case of $T=100, N=200$-there is an overall reduction in the MSFE, pointing to an increase in the predictive ability across all the methods. This suggests that a higher ratio of $N$ to $T$ is better. This is because a larger cross-sectional dimension is helpful for more precise estimation of the underlying factor and thereby leads to better predictive performance.

When dealing with high-dimensional data sets, the substantial heterogeneity in the data may be accompanied by different degrees of instabilities contained in the predictors $\mathbf{x}_{t}$. Therefore, we repeat these simulation exercises along the lines of Bates et al. (2013) and let only a subset $J$ of variables, randomly selected from a uniform distribution, exhibit regime-switching factor loadings. The lower part of Table 1 reports the case where the share of variables experiencing instabilities in their loadings is 0.25 . The results are relatively similar to the ones obtained with all the variables experiencing instabilities in the loadings in that the MSS-3PRF obtains the best forecasting performance. However, the method showing the second-best forecasting performance is TPCA. Another important difference is that the increase in the predictive ability of the methods when $N$ increases and $T$ remains constant is not systematically observed through all the scenarios under consideration, as was the case where all the variables were experiencing instabilities in the loadings. This is simply because in this case there is less cross-sectional information that can help to capture the non-linearities in the time dimension of the data. We performed the same simulation exercise when the share of variables experiencing instabilities in the loadings is 0.50 and 0.75. The results remained qualitatively unchanged. To conserve space, we do not report the results for these DGPs, but these results are available upon request.

The results reported in Table 1 were based on a DGP where the factor loadings associated with each predictor variable were driven by their own Markov chain, and these Markov chains were assumed to experience a high degree of interdependence between them to mimic the behaviour that macroeconomic and financial data usually tend to exhibit. However, we are interested in assessing the performance of the methods when the assumption of interdependent Markov chains is no longer valid - that is, when the loadings depend on Markov chains that are independent of each other. The upper part of Table 2 reports the MSFE for the case where factor loadings are driven by independent Markov chains. The results provide the same consistent message obtained from the previous exercises: the MSS-3PRF shows the most accurate predictive performance among the methods under consideration, followed by the MS-3PRF. These results imply that regardless of the relationship between 
the Markov chains driving the factor loadings, the non-linear methods proposed in this paper consistently outperform the competing linear methods.

Finally, we compare the forecasting performance of the methods under consideration when the dynamics of the factor are also subject to regime changes. In particular, we repeat the Monte Carlo exercises assuming that the DGP of the factor is given by

$$
f_{t}=\rho_{f, t} f_{t-1}+u_{f, t}
$$

where the dynamics of the autoregressive coefficient depend on the seed Markov chain,

$$
\rho_{f, t}=\rho_{1 f} S_{0, t}+\rho_{0 f}\left(1-S_{0, t}\right)
$$

The lower part of Table 2 shows the corresponding MSFE for the different scenarios, showing that the MSS-3PRF and the MS-3PRF perform better than the linear benchmarks in most of the cases. Notice that, in this case, the MS-3PRF model is not correctly specified given that there is no regime switching in the factor loadings.

Overall, conditional on our DGPs, we can conclude that, on average, the MSS-3PRF is the framework best able to capture instabilities in the relationship between the set of predictors and its common factor, followed by the MS-3PRF. Both non-linear frameworks outperform linear approaches. Regarding the linear frameworks, in general, TPCA outperforms the $3 \mathrm{PRF}$ and the PC-LARS, and PCA obtains the weakest forecasting performance.

\section{Empirical Applications}

The first application is related to exchange rate forecasting. This is highly relevant given that it has long been recognized that non-linearities play an important role in the dynamics of exchange rates (see, e.g., the early contribution in Chinn (1991) and more recently Rossi (2013) and Abbate and Marcellino (2016)). However, it is only recently that the literature on exchange rate forecasting has concentrated on the role and importance of factors for predicting exchange rates (see, e.g., Engel et al. (2015) in a linear context). Putting the MS-3PRF approach to work in the context of exchange rate forecasts is highly relevant: it allows us to combine the non-linear dynamics observed in exchange rate movements with the factor structure driving systematic variations in exchange rates, which has recently gained traction in the exchange rate forecasting literature. Our second empirical application is a standard macroeconomic forecasting application in that we use the McCracken and $\mathrm{Ng}$ (2015) data set to forecast economic activity in the United States. 


\subsection{Forecasting exchange rates}

In this first forecasting exercise, we construct factors from a cross-section of nominal bilateral U.S. dollar (USD) exchange rates against a panel of 26 currencies. We extract factors from the MS-3PRF, MSS-3PRF, linear 3PRF, PCA, TPCA and PC-LARS. We then use the resulting factors to forecast selected bilateral exchange rates. (All currency pairs use the USD as numéraire.) The choice of the data set draws from the exercise in GreenawayMcGrevy et al. (2016). The data set is monthly, and the full sample size extends from January 1995 to December 2015. The data are obtained from the International Financial Statistics of the International Monetary Fund, and the monthly data are taken as the monthly average of daily data. The data set consists of the currencies of Australia (AUS), ${ }^{7}$ Brazil (BRA), Canada (CAN), Chile (CHI), Columbia (COL), the Czech Republic (CZE), the euro (EUR), Hungary (HUN), Iceland (ICE), India (IND), Israel (ISR), Japan (JPN), Korea (KOR), Mexico (MEX), Norway (NOR), New Zealand (NZE), the Philippines (PHI), Poland (POL), Romania (ROM), Singapore (SIN), South Africa (RSA), Sweden (SWE), Switzerland (SUI), Taiwan (TAI), Turkey (TUR) and the United Kingdom (GBR). ${ }^{8}$

We consider forecast horizons, $h$, ranging from 1 month to 12 months and report predictive results for selected major currencies: the euro, the British pound, the Japanese yen and the Canadian dollar. The first estimation sample runs from February 1995 to March 2007, and it is recursively expanded until we reach the end of the estimation sample. Hence, the forecast evaluation period extends from April 2007 to December 2015. As in the Monte Carlo experiment, we compare the forecasts obtained from the MS-3PRF with forecasts derived from PCA, linear 3PRF, TPCA and PC-LARS. Moreover, we use two different versions of the MS-3PRF, one with regime switching only in the first step (i.e., in the factor loadings), and one with regime-switching in the first and third steps (i.e., in the factor loadings and in the parameters of the forecasting equation). In the first step of the algorithm for the MS-3PRF approach, we model regime changes in all parameters of the model (i.e., intercept, slope and innovation variance), since we obtained stronger fit -as measured by the Schwarz information criterion (SIC) - with such a specification. Note also that we consider a model with two factors across all methods. The choice of the number of factors follows the modelling choices in Engel et al. (2015), Greenaway-McGrevy et al. (2016) and Verdelhan (2015), but, qualitatively, our results are robust to the use of one or three factors in the predictive equation. We also include the MSS-3PRF approaches in the

\footnotetext{
${ }^{7}$ The three-letter country codes follow the convention from the International Olympic Committee except for Taiwan, labelled as TAI.

${ }^{8}$ Data for the euro before January 1999 and Taiwan were obtained from the U.S. Federal Reserve G.5 table (monthly average of daily data).
} 
set of models we consider (both versions - that is, with regime switches in the first pass only and with regime switches in the first and third passes).

All exchange rate series are taken as the first difference of their logarithm before performing factor analysis. In the case of the $3 \mathrm{PRF}$ approaches, we use the automatic proxyselection procedure from Kelly and Pruitt (2015); that is, we use the exchange rate we are interested in forecasting as a target proxy when extracting the first factor and then proceed sequentially as outlined in Table 2 from Kelly and Pruitt (2015). For PCA, TPCA and PC-LARS, we standardize the data recursively in the estimation before estimating the factors. In contrast, the $3 \mathrm{PRF}$ approaches do not require standardizing the data before estimation.

For the prediction step, in the linear cases - that is, for PCA, TPCA, PC-LARS, 3PRF, MS-3PRF (first pass) and MSS-3PRF (first pass) - the $h$-period-ahead forecasts for a specific currency $R_{t+h \mid t}^{j}$ are constructed in level based on the following equation:

$$
R_{t+h \mid t}^{j}=R_{t}^{j}\left(1+\hat{\alpha}+\boldsymbol{F}_{\boldsymbol{t}}^{\prime} \hat{\boldsymbol{\beta}}\right)
$$

where $\hat{\alpha}$ and $\hat{\boldsymbol{\beta}}$ are obtained from the following regression (for simplicity of the notation, we omit $h$ subscripts from the coefficients $\alpha$ and $\boldsymbol{\beta}$ ):

$$
\Delta r_{t, h}^{j}=\alpha+\boldsymbol{F}_{t-h}^{\prime} \boldsymbol{\beta}+\epsilon_{t}
$$

where $\Delta r_{t, h}^{j}$ indicates the $h$-period change in the logarithm of the exchange rate $R_{t}^{j}$ (i.e., $\left.\Delta r_{t, h}^{j}=\ln \left(R_{t}^{j}\right)-\ln \left(R_{t-h}^{j}\right)\right)$, and $\boldsymbol{F}_{t}$ indicates the factors extracted from either PCA, TPCA, PC-LARS, 3PRF or MS-3PRF approaches. In the case of the MS-3PRF (first and third passes) and MSS-3PRF (first and third passes), equation 24 is modified as follows:

$$
\Delta r_{t, h}^{j}=\alpha\left(S_{t}^{j}\right)+\boldsymbol{F}_{t-h}^{\prime} \boldsymbol{\beta}\left(S_{t}^{j}\right)+\epsilon_{t}\left(S_{t}^{j}\right)
$$

where $S_{t}^{j}$ is a two-regime Markov chain, distinct across all predicted currencies $j$, with constant transition probabilities. In the case of the MS-3PRF (first and third passes), as is commonly done in forecasting exercises with Markov-switching models, the forecasts are calculated as a weighted average of forecasts conditional on the parameters being in a given regime. The predicted probabilities of being in a given regime $k, h$ periods ahead, are obtained recursively as

$$
P\left(S_{t+h \mid t}^{j}=k\right)=\sum_{i=1}^{2} p_{i k}^{j} P\left(S_{t+h-1 \mid t}^{j}=k\right),
$$

where $p_{i k}^{j}$ indicates the constant transition probabilities, and 2 is the total number of regimes. 
In the case of the MSS-3PRF (first and third passes) approach, the forecasts are calculated conditional on being in a given regime; that is, the predicted probabilities are obtained as

$$
P\left(S_{t+h \mid t}^{j}=k\right)=I\left(P\left(S_{t \mid t}^{j}=k\right)\right)
$$

where $I(\cdot)$ is an indicator variable that indicates the regimes with the highest smoothed probability at the origin of the forecast horizon. As such, this corresponds to the approach often used to plot (regime-specific) impulse responses in MS-VAR models (see, e.g., Hubrich and Tetlow (2015)).

As an illustration of the MS-3PRF approach to extract factors from a panel of exchange rates, Figure 1 reports the Markov-switching factor loadings based on the MS-3PRF approach using the Canadian dollar as a target proxy, that is, the factor loadings associated with the first factor. From this figure, one can see that there is substantial time variation in factor loadings for a number of currencies (e.g., the Australian dollar and the New Zealand dollar), whereas for other currencies, there is little time variation in the factor loadings (e.g., the euro and the Swiss franc).

A number of additional comments are in order. First, we do not use observable factors to model the dynamics of exchange rates. Verdelhan (2015) finds that exchange rate variations are driven by a two-factor structure: a U.S.-dollar factor that serves as a proxy for global macroeconomic risk and a carry factor that is interpreted as capturing uncertainty risk. Our analysis differs from this study in that our focus is on out-of-sample predictive ability. Hence, we do not aim to provide a structural interpretation to the factors we extract.

Table 3 reports point forecasting results for specific currencies: the Canadian dollar (CAD), the euro (EUR), the Japanese yen (JPY) and the British pound (GBP), all relative to the USD. These are G7 currencies, and among the most traded currency pairs according to the Bank for International Settlements Triennal Central Bank Survey. ${ }^{9}$ The point forecast results are presented as the MSFE of a specific approach relative to the MSFE obtained from the no-change forecast. The no-change forecast is the standard benchmark in the exchange rate forecasting literature (see, e.g., Rossi (2013)). This table also reports the results of the Diebold and Mariano (1995) test of equal out-of-sample predictive accuracy using the no-change forecast as a benchmark. ${ }^{10}$ First, the models' forecasting performance relative to the no-change forecast is typically the strongest for forecast horizon $h=1$ (except for the JPY/USD). The improvement in forecast accuracy relative to the random walk

\footnotetext{
${ }^{9}$ See http://www.bis.org/publ/rpf $x 16 f x$.pdf.

${ }^{10}$ The Diebold and Mariano (1995) test of equal out-of-sample predictive accuracy is reported to give a sense of statistical significance of the point forecasting results. However, this test is based on the population MSFE (not the actual MSFE), so this test tends to reject the null of equal MSFEs too often.
} 
is also statistically significant according to the Diebold and Mariano test of equal MSFE when forecasting the Canadian dollar at forecast horizon $h=1$ across most approaches (this is also true to a lesser extent for the British pound). Second, the PC-LARS approach performs best for forecast horizon $h=1$ when forecasting the euro and the British pound, albeit it is closely followed by PCA and TPCA in those cases. Third, for the Canadian dollar and the Japanese yen, modelling time variation in the forecasting equation is relevant in that this leads to substantial forecasting improvement over the no-change forecast at distant forecast horizons $h=\{9,12\}$ for the Japanese yen and $h=\{2,3,6,9,12\}$ for the Canadian dollar, using the MS-3PRF (first and third passes) approach. ${ }^{11}$

Next, Table 4 shows the directional accuracy forecasting results, which are broadly in line with the point forecast results. Under the null hypothesis of no directional accuracy, one would expect a success ratio of 0.5 . In this table, we also report the results of the Pesaran and Timmermann (2009) test to evaluate the statistical significance of the directional accuracy results. Across all forecasting approaches, the success ratios tend to be stronger for forecast horizon $h=1$, except for the JPY/USD. It is also interesting to note that the success ratios are especially strong at distant forecast horizons for selected currencies, as high as 67.3 per cent for the CAD/USD and 79.7 per cent for the JPY/USD in the case of the MS-3PRF and MSS-3PRF with regime changes in the first and third passes.

\subsection{Forecasting economic activity}

In this application, we use the McCracken and $\mathrm{Ng}$ (2015) data set to forecast eight major quarterly U.S. variables: GDP, consumption, investment, exports, imports, total hours, GDP inflation and personal consumption expenditures (PCE) inflation. ${ }^{12}$ We implemented the following outlier corrections to the predictors: observations of the transformed series with absolute median deviations larger than six times the inter-quartile range were replaced with the median value of the preceding five observations. The full sample extends from the third quarter of 1960 to the third quarter of 2015. In the forecasting exercise, the first estimation sample extends from the third quarter of 1960 to the fourth quarter of 1984, and it is expanded recursively until we reach the end of the sample. We consider forecast horizons, $h$, ranging from one quarter to eight quarters. We use eight competing

\footnotetext{
${ }^{11}$ Admittedly, in the case of the euro, the forecasting performance of the MS-3PRF (first and third passes) and the MSS-3PRF (first and third passes) approaches deteriorates as the forecast horizon lengthens, suggesting that it is not always relevant to model regime shifts in the forecasting equation.

${ }^{12}$ Data descriptions and details on data transformation are available online at https://research. stlouisfed.org/econ/mccracken/fred-databases/Appendix_Tables_Update.pdf. The slight modifications we made to the original data set are reported in the appendix.
} 
approaches: PCA from which we extract five factors from the underlying data set, although we use only the first one in the forecasting equation; PCA where hard thresholding has been performed before extracting the first principal component to forecast (TPCA); PCA where soft thresholding has been performed before extracting the first principal component to forecast (PC-LARS); linear 3PRF; MS-3PRF and MSS-3PRF with regime-switching parameters in the first pass only; and MS-3PRF and MSS-3PRF with regime-switching parameters in the first and third passes. For the $3 \mathrm{PRF}$ approaches, we use one factor and use the predicted variable as a target proxy in the first step of the 3PRF approach (target-proxy $3 \mathrm{PRF}) .^{13}$

We first report results from an in-sample exercise. Figure 2 shows the estimated factors across all six methods (PCA, TPCA, PC-LARS, 3PRF, MS-3PRF and MSS-3PRF; the latter three methods use GDP growth as a target variable). This shows that the factor estimates are relatively similar across approaches and that they closely follow the U.S. business cycle. As in McCracken and Ng (2015), we calculate diffusion indexes $\left(\hat{F}_{t}\right)$ based on the partial sums of the factor estimates $\hat{f}_{t}$; that is, $\hat{F}_{t}=\sum_{j=1}^{t} \hat{f}_{j}$. (The reason for doing so is that diffusion indexes summarize information contained in the trend as opposed to the "raw" factors that are estimated on stationary data, so the resulting factors are too volatile for turning point analysis.) The factors $f_{t}$ are extracted with the six aforementioned methods. We then implemented the Bry and Boschan (1971) algorithm to estimate expansions and recessions from these diffusion indexes. ${ }^{14}$ The resulting classification of U.S. business cycles obtained from the MS-3PRF diffusion index has the strongest correlation with the National Bureau of Economic Research dummy variable of expansions and recessions (0.563) followed by the 3PRF diffusion index (0.543), MSS-3PRF diffusion index (0.506), PC-LARS diffusion index (0.497), PCA diffusion index (0.486) and TPCA diffusion index (0.467). Moreover, only the MS-3PRF and MSS-3PRF approaches obtain a perfect classification of recessions, while PCA, TPCA and 3PRF diffusion indexes have a near perfect classification of recessions (these three methods identify the 1973-1974 recession with a one-quarter lag). This suggests that the MS-3PRF approach has important information related to the state of the business cycle that is not necessarily reflected in competing approaches.

\footnotetext{
${ }^{13}$ When estimating the number of factors using information criteria, it is common to find a large number of factors summarizing the comovements of U.S. macroeconomic variables (e.g., McCracken and $\mathrm{Ng}$ (2015) estimate eight factors in the FRED-MD monthly macroeconomic database). However, in the forecasting exercise, in line with the literature, we use the first factor in the predictive equation. This corresponds to a real economic activity factor that closely follows the U.S. business cycle dynamics (see Figure 2). Using the first two factors in the predictive equation led to little changes in the forecasting performance.

${ }^{14}$ The Bry and Boschan (1971) algorithm is a non-parametric method to estimate cycles in time series. We implemented the quarterly version of the Bry and Boschan (1971) algorithm from Harding and Pagan (2002), using the GAUSS code available at http://www.ncer.edu.au/resources/data-and-code.php.
} 
An attractive feature of Markov-switching models is their ability to endogenously estimate regimes. Figure 3 shows a heat map of the smoothed probability of being in the first regime (associated with adverse business cycle conditions) for the in-sample factor loadings obtained from the first step of the MS-3PRF approach. First, it is interesting to note that, across all series, there is substantial time variation in the smoothed probability, suggesting that there is evidence in favour of regime shifts in the factor loadings. Second, the timing of the shifts in the factor loadings coincides with the changes in business cycle phases for a large number of series (e.g., output and income, as well as labour market variables). Additional evidence on regime shifts in the factor loadings is provided in Figure 4. This figure shows that there is substantial variation in the factor loadings for the unemployment rate and industrial production related to the state of the business cycle. Selected financial and credit variables (S\&P 500 returns and consumer loans) also exhibit substantial time variation, suggesting that the assumption of constant factor loadings often employed with this type of data set is likely to be too restrictive.

A few additional comments related to the out-of-sample forecasting exercise are required. First, note that macroeconomic variables are typically subject to substantial revisions and different publication lags. In this empirical exercise, we abstract from this issue and consider revised data. While this is not a fully realistic approach from a practitioner's perspective, there is no reason to think that one specific approach would benefit more from this simplification. Hence, this remains a useful forecasting exercise to compare the relative merits of each forecasting approach. Second, across all approaches, quarterly factors are extracted from the monthly data set of McCracken and $\mathrm{Ng}$ (2015), where quarterly data are taken as quarterly averages of monthly data before performing factor analysis. Obviously, alternative temporal aggregation schemes could be adopted, but we found that the in-sample correlation of the real activity factor was very strong compared with a situation where one would use the last monthly observation of the quarter as a quarterly observation before performing factor analysis (about 0.95 between these two aggregation schemes across the different factor approaches). ${ }^{15}$ Our temporal aggregation scheme is standard in the literature (see, e.g., section 6.1 in Stock and Watson (2016)), and we leave the issue of a mixed-frequency setting to future research. ${ }^{16}$ Third, the forecasts are constructed as

\footnotetext{
${ }^{15}$ This result still holds when doing PCA at a monthly frequency and then aggregating the factor at a quarterly frequency.

${ }^{16}$ As a side note, the first pass of the 3PRF filter could possibly accommodate mixed-frequency data using the techniques outlined in Foroni et al. (2015); whereas, in the third pass of the filter, unrestricted mixed data sampling (MIDAS) polynomials could be used as in Hepenstrick and Marcellino (2016), and regime-switching parameters in the mixed-frequency predictive equation could be modelled as in Guérin and Marcellino (2013).
} 
follows:

$$
y_{t+h \mid t}=\hat{\alpha}+\hat{\beta}(L) \hat{f}_{t}+\hat{\gamma}(L) y_{t}
$$

where $\beta(L)$ and $\gamma(L)$ are finite-order lag polynomials, whose lag lengths are obtained with the SIC at the beginning of the forecasting exercise, using a maximum lag length of 6 for $\gamma(L)$ and 3 for $\beta(L)$. All predicted variables $y_{t}$ are taken as the first difference of their logarithm. For the MS-3PRF and MSS-3PRF with switches in the first and third passes, we consider regime-switching parameters in all parameters of equation 28 and in the variance of the error term.

Tables 5 and 6 show the out-of-sample forecasting results. All results are reported relative to the forecasts obtained from PCA. Hence, a number below 1 indicates that a given approach outperforms PCA. We also report the results of the Diebold and Mariano (1995) test of equal out-of-sample predictive accuracy using PCA as a benchmark. Overall, across all forecast horizons and predicted variables (64 cases), the MS-3PRF and MSS-3PRF obtain the best forecasting results in 35 cases, the linear 3PRF in 16 cases, PC-LARS in 10 cases and TPCA in 1 case. In the remainder of the cases, PCA performs best. It is interesting to note that the MSS-3PRF (first and third passes) approach performs best for forecasting inflation (both PCE inflation and GDP inflation), and it does so significantly according to the Diebold and Mariano (1995) test at long forecast horizons (i.e., for $h>4$ for GDP inflation and $h>3$ for PCE inflation). The rationale for the strong forecasting performance of the MSS-3PRF (first and third passes) when forecasting inflation at distant forecast horizons is that over the full sample we look at, there is a decline in the rate of inflation. Moreover, as the forecast horizon increases, forecasts tend to converge toward the full sample mean of inflation. However, forecasts from the MSS-3PRF (first and third passes) approach give zero weight to the high inflation observed in the early part of the sample as opposed to the forecasts from the competing approaches, which necessarily include information from high inflation episodes at distant forecast horizons. When forecasting aggregate economic activity (GDP), the MSS-3PRF approach performs best at short forecast horizons while the linear 3PRF tends to perform best for distant forecast horizons, and the improvements in forecast accuracy relative to PCA are typically statistically significant according to the Diebold and Mariano (1995) test. The MS-3PRF forecasting approaches perform particularly well relative to PCA when predicting export, import and hours worked, whereas PC-LARS performs well for forecasting investment and hours worked at relatively short forecast horizons (i.e., $h<4$ ). 


\section{Conclusion}

In this paper, we extended the linear three-pass regression filter to settings where parameters can vary according to Markov processes, introducing the Markov-switching three-pass regression filter. A key advantage of our framework is to circumvent the computational difficulties associated with the estimation of a large-scale dynamic factor model with regimeswitching parameters without foregoing flexibility in modelling choices.

In both simulation and empirical examples, our method compares favourably with existing alternatives in terms of forecasting performance. The MS-3PRF approach is also attractive beyond forecasting applications. For example, the MS-3PRF approach would easily allow one to model regime-switching correlations often observed in finance in a highdimensional setting. This could be relevant in the context of the growing literature aiming at measuring network connectedness among financial firms or asset classes (see, e.g., Billio et al. (2012) or Diebold and Yilmaz (2014)). Likewise, the MS-3PRF framework could be used in the context of structural factor-augmented VAR models that are commonly used in macroeconomics. Overall, thanks to its generality and ease of implementation, the MS3PRF approach offers a promising framework to model regime changes in high-dimensional settings for a large class of applications in macroeconomics and finance. 


\section{References}

Aastveit, K., Carreiro, A., Clark, T., and Marcellino, M. (2016). Have standard VARs remained stable since the crisis? Journal of Applied Econometrics, forthcoming.

Abbate, A. and Marcellino, M. (2016). Modelling and forecasting exchange rates with time-varying parameter models. Journal of the Royal Statistical Society, Series A, forthcoming.

Amengual, D. and Watson, M. W. (2007). Consistent Estimation of the Number of Dynamic Factors in a Large N and T Panel. Journal of Business 83 Economic Statistics, 25:91-96.

Bai, J. and Ng, S. (2008). Forecasting economic time series using targeted predictors. Journal of Econometrics, 146(2):304-317.

Barnett, W. A., Chauvet, M., and Leiva-Leon, D. (2016). Real-time nowcasting of nominal GDP with structural breaks. Journal of Econometrics, 191(2):312-324.

Bates, B. J., Plagborg-Moller, M., Stock, J. H., and Watson, M. W. (2013). Consistent factor estimation in dynamic factor models with structural instability. Journal of Econometrics, 177(2):289-304.

Billio, M., Getmansky, M., Lo, A. W., and Pelizzon, L. (2012). Econometric measures of connectedness and systemic risk in the finance and insurance sectors. Journal of Financial Economics, 104(3):535-559.

Bry, G. and Boschan, C. (1971). Cyclical Analysis of Time Series: Procedures and Computer Programs. National Bureau of Economic Research.

Camacho, M., Pérez-Quirós, G., and Poncela, P. (2012). Markov-switching dynamic factor models in real time. CEPR Discussion Papers 8866, C.E.P.R. Discussion Papers.

Canova, F. (1993). Modelling and Forecasting Exchange Rates with a Bayesian TimeVarying Coefficient Model. Journal of Economic Dynamics and Control, 17:233-261.

Carter, A. and Steigewald, D. (2012). Testing for Regime Switching: A Comment. Econometrica, 80:1809-1812.

Chauvet, M. (1998). An Econometric Characterization of Business Cycle Dynamics with Factor Structure and Regime Switching. International Economic Review, 39(4):969-96.

Cheng, X., Liao, Z., and Schorfheide, F. (2016). Shrinkage Estimation of High-Dimensional Factor Models with Structural Instabilities. Review of Economic Studies, forthcoming. 
Ching, W.-K., Fung, E., and Ng, M. (2002). A multivariate Markov chain model for categorical data sequences and its applications in demand predictions. IMA Journal of Management Mathematics, 13:187-199.

Chinn, M. D. (1991). Some linear and nonlinear thoughts on exchange rates. Journal of International Money and Finance, 10(2):214-230.

Cho, J. S. and White, H. (2007). Testing for Regime Switching. Econometrica, 75:16711720 .

D’Agostino, A., Gambetti, L., and Giannone, D. (2013). Macroeconomic forecasting and structural change. Journal of Applied Econometrics, 28(1):82-101.

De Mol, C., Giannone, D., and Reichlin, L. (2008). Forecasting using a large number of predictors: Is Bayesian shrinkage a valid alternative to principal components? Journal of Econometrics, 146(2):318-328.

Del Negro, M. and Otrok, C. (2008). Dynamic factor models with time-varying parameters: measuring changes in international business cycles. Staff Reports 326, Federal Reserve Bank of New York.

Diebold, F. X. and Mariano, R. S. (1995). Comparing Predictive Accuracy. Journal of Business \& Economic Statistics, 13(3):253-63.

Diebold, F. X. and Yilmaz, K. (2014). On the network topology of variance decompositions: Measuring the connectedness of financial firms. Journal of Econometrics, 182(1):119-134.

Douc, R., Moulines, E., and Rydén, T. (2004). Asymptotic properties of the maximum likelihood estimor in autoregressive models with Markov regime. Annals of Statistics, $32(5): 2254-2304$.

Efron, B., Hastie, T., Johnstone, I., and Tibshirani, R. (2004). Least Angle Regression. Annals of Statistics, 32(2):407-499.

Eickmeier, S., Lemke, W., and Marcellino, M. (2015). Classical time varying factoraugmented vector auto-regressive modelsestimation, forecasting and structural analysis. Journal of the Royal Statistical Society Series A, 178(3):493-533.

Engel, C., Mark, N. C., and West, K. D. (2015). Factor Model Forecasts of Exchange Rates. Econometric Reviews, 34(1-2):32-55.

Forni, M., Hallin, M., Lippi, M., and Reichlin, L. (2005). The Generalized Dynamic Factor Model: One-Sided Estimation and Forecasting. Journal of the American Statistical Association, 100:830-840. 
Foroni, C., Guérin, P., and Marcellino, M. (2015). Using low frequency information for predicting high frequency variables. Norges Bank Working Paper, 2015/13.

Greenaway-McGrevy, R., Mark, N., Sul, D., and Wu, J.-L. (2016). Identifying Exchange Rate Common Factors. Mimeo Notre Dame.

Groen, J. and Kapetanios, G. (2016). Revisiting useful approaches to data-rich macroeconomic forecasting. Computational Statistics \& Data Analysis, 100:221-239.

Guérin, P. and Leiva-Leon, D. (2016). Monetary Policy, Stock Market and Sectoral Comovement. Mimeo.

Guérin, P. and Marcellino, M. (2013). Markov-Switching MIDAS Models. Journal of Business \& Economic Statistics, 31(1):45-56.

Hamilton, J. D. and Owyang, M. T. (2012). The Propagation of Regional Recessions. The Review of Economics and Statistics, 94(4):935-947.

Harding, D. and Pagan, A. (2002). Dissecting the cycle: a methodological investigation. Journal of Monetary Economics, 49(2):365-381.

Hepenstrick, C. and Marcellino, M. (2016). Forecasting with Large Unbalanced Datasets: The Mixed-Frequency Three-Pass Regression Filter. SNB Working Papers, 2016-04.

Hubrich, K. and Tetlow, R. J. (2015). Financial stress and economic dynamics: The transmission of crises. Journal of Monetary Economics, 70(C):100-115.

Kelly, B. and Pruitt, S. (2015). The three-pass regression filter: A new approach to forecasting using many predictors. Journal of Econometrics, 186(2):294-316.

Leroux, B. (1992). Maximum-likelihood estimation for hidden Markov models. Stochastic Processes and their Applications, 40:127-143.

McCracken, M. W. and Ng, S. (2015). FRED-MD: A Monthly Database for Macroeconomic Research. Journal of Business $\&$ Economic Statistics, forthcoming.

Mikkelsen, J. G., Hillebrand, E., and Urga, G. (2015). Maximum Likelihood Estimation of Time-Varying Loadings in High-Dimensional Factor Models. CREATES Research Papers 2015-61, School of Economics and Management, University of Aarhus.

Pesaran, M. H. and Timmermann, A. (2009). Testing Dependence Among Serially Correlated Multicategory Variables. Journal of the American Statistical Association, 104(485):325-337. 
Rossi, B. (2013). Exchange Rate Predictability. Journal of Economic Literature, 51(4):1063-1119.

Sims, C. (1993). A 9 Variable Probabilistic Macroeconomic Forecasting Model. Business Cycles, Indicators and Forecasting, NBER studies in business cycles, 28:179-214.

Sims, C. A. and Zha, T. (2006). Were There Regime Switches in U.S. Monetary Policy? American Economic Review, 96(1):54-81.

Smith, A., Prasad, N., and Tsai, C.-L. (2006). Markov-switching Model Selection using Kullback-Leibler Divergence. Journal of Econometrics, 134:553-577.

Stock, J. and Watson, M. (2002a). Forecasting Using Principal Components From a Large Number of Predictors. Journal of the American Statistical Association, 97:1167-1179.

Stock, J. H. and Watson, M. W. (2002b). Macroeconomic Forecasting Using Diffusion Indexes. Journal of Business \& Economic Statistics, 20(2):147-62.

Stock, J. H. and Watson, M. W. (2016). Factor Models and Structural Vector Autoregressions in Macroeconomics. Mimeo.

Verdelhan, A. (2015). The Share of Systematic Variation in Bilateral Exchange Rates. Journal of Finance, forthcoming.

Von Ganske, J. (2016). A Regime Switching Partial Least Squares Approach to Forecasting Industry Stock Returns. Mimeo Edhec-Risk Institute. 


\section{Appendix}

\section{A.1 Description of the hard-thresholding forecasting approach}

The hard-thresholding algorithm consists of the following steps (this description stems partly from Bai and Ng (2008)):

1. For each variable $x_{i, t}$, perform a time series regression of the variable to forecast $y_{t}$ on $x_{i, t}$ and a constant. Let $t_{i}$ denote the t-statistic associated with $x_{i, t}$.

2. Let $k_{\alpha}^{*}$ be the number of series whose $\left|t_{i}\right|$ exceeds a threshold significance level, $\alpha$. In our application, we use a threshold of 1.65 , which corresponds to a one-sided 5 per cent significance level for the t-test.

3. Let $\chi_{t}(\alpha)=\left(x_{[1 t]}, \ldots, x_{\left[k_{\alpha}^{*}\right]}\right)$ be the corresponding set of predictors. Estimate $f_{t}$ from $\chi_{t}(\alpha)$ by the method of principal component.

4. Estimate equation 28 to calculate the $h$-period-ahead forecast $y_{t+h}$.

This approach is denoted as TPCA.

\section{A.2 Description of the soft-thresholding forecasting approach}

The soft-thresholding approach we adopt follows from the least angle regressions (LARS) method described in Bai and $\mathrm{Ng}$ (2008). In detail, we select the set of the first $K$ predictors $x_{i, t}$ selected by forward stagewise selection regressions to extract principal component(s). In the macroeconomic forecasting application, we use $K=30$ predictors, since it is the number of predictors retained by Bai and Ng (2008) and Kelly and Pruitt (2015) when forecasting macroeconomic variables with a data set similar to ours. For the exchange rate forecasting application, we retain the first $K=10$ predictors ordered by LARS to extract principal components, which corresponds to slightly more than a third of the total number of predictors (26). Finally, in the Monte Carlo experiments, we set $K=30$ across all DGPs.

This approach is denoted as $P C-L A R S$. 


\section{A.3 Additional details on the macroeconomic forecasting exer- cise}

In the macroeconomic forecasting empirical application, we use the May 2016 vintage of the McCracken and $\mathrm{Ng}$ (2015) data set as available online at https://research.stlouisfed. org/econ/mccracken/fred-databases/. We use the exact same transformation as suggested by McCracken and $\mathrm{Ng}$ (2015). However, due to missing observations, we omit the following five series in our analysis (FRED mnemonics are in parentheses): "New Orders for Consumer Goods" (ACOGNO), "New Orders for Nondefense Capital Goods" (ANDENOx), "Trade-weighted U.S. Dollar Index: Major Currencies" (TWEXMMTH), "Consumer Sentiment Index" (UMCSENTx) and the "VXO" (VXOCLSx).

The eight variables we forecast in the macroeconomic forecasting application are Gross Domestic Product (GDPQ@USNA), Personal Consumption Expenditures (CQ@USNA), Gross Private Domestic Investment (IQ@USNA), Exports of Goods \& Services (XQ@USNA), Imports of Goods \& Services (MQ@USNA), Business Sector: Hours of All Persons (LXBH@USECON), Gross Domestic Product: Chain Price Index (JGDP@USNA) and Personal Consumption Expenditures: Chain Price Index (JC@USNA). (Haver Analytics mnemonics are in parentheses.) 
Figure 1: Markov-switching factor loadings - Canadian dollar as a target proxy

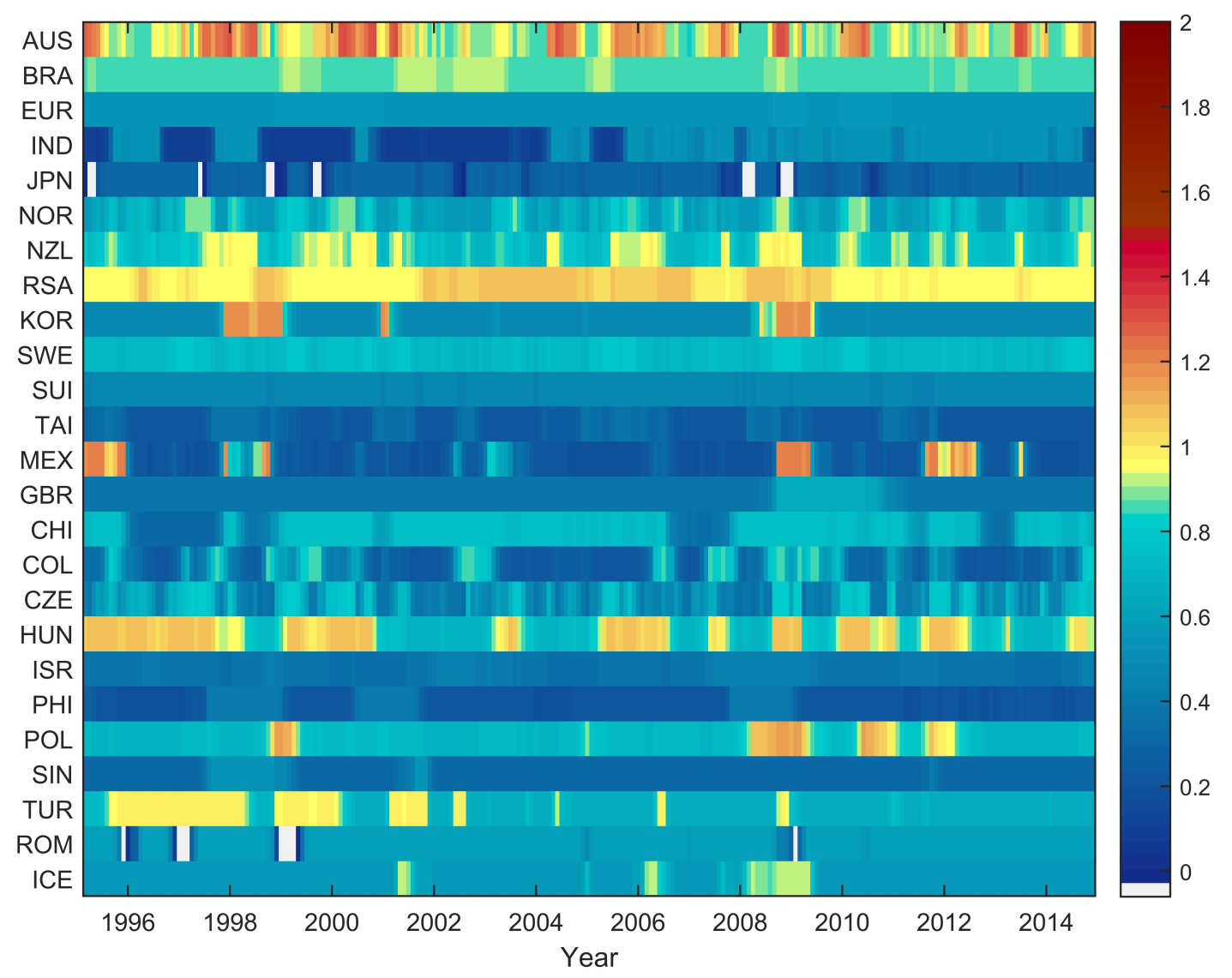

Note: Dark red indicates higher values for the factor loadings obtained with the MS-3PRF approach with the Canadian dollar as a target proxy. 


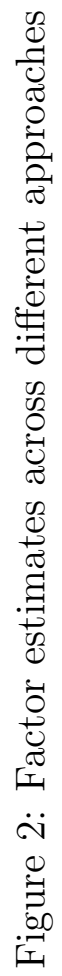

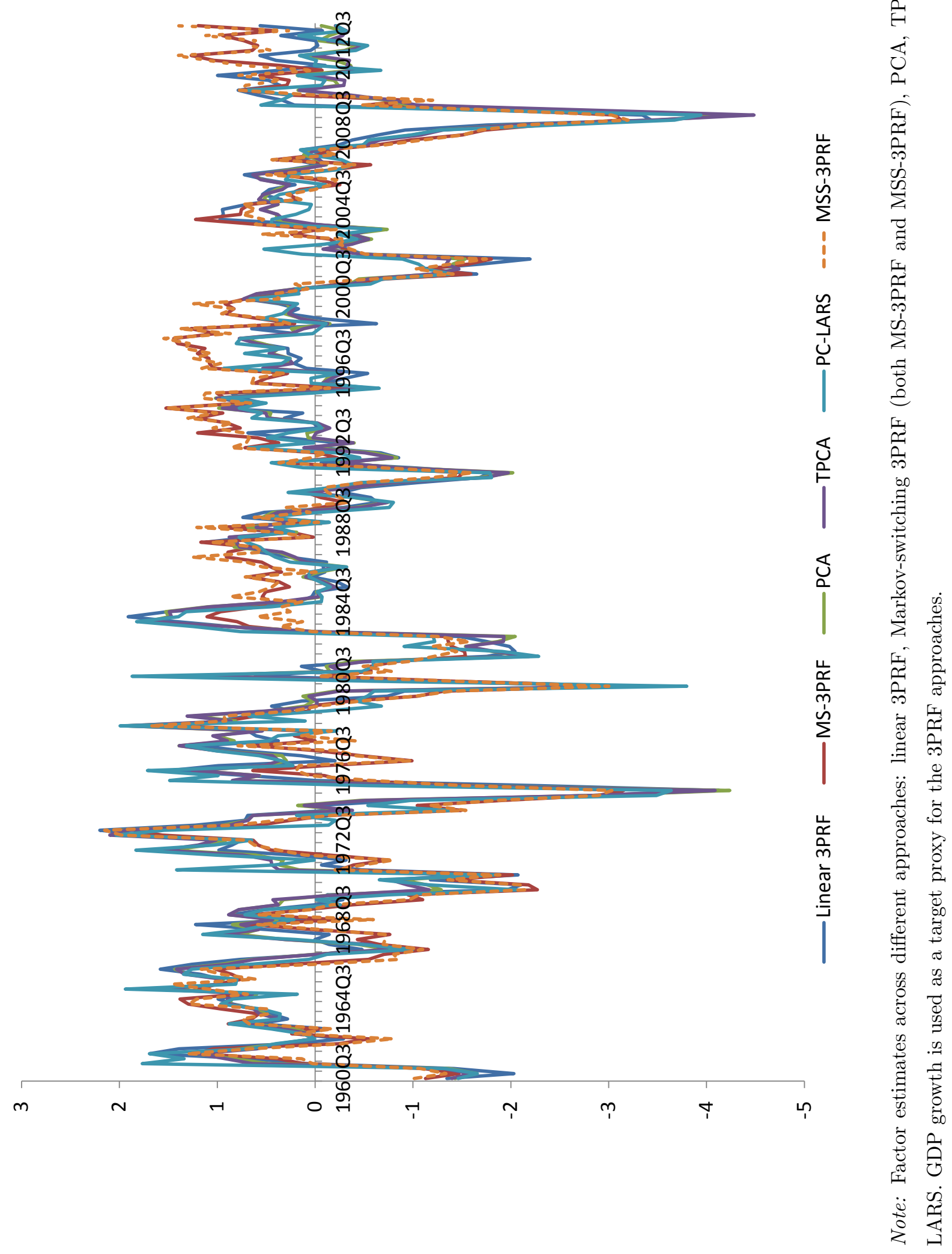


Figure 3: Probability of being in the first regime for the factor loadings

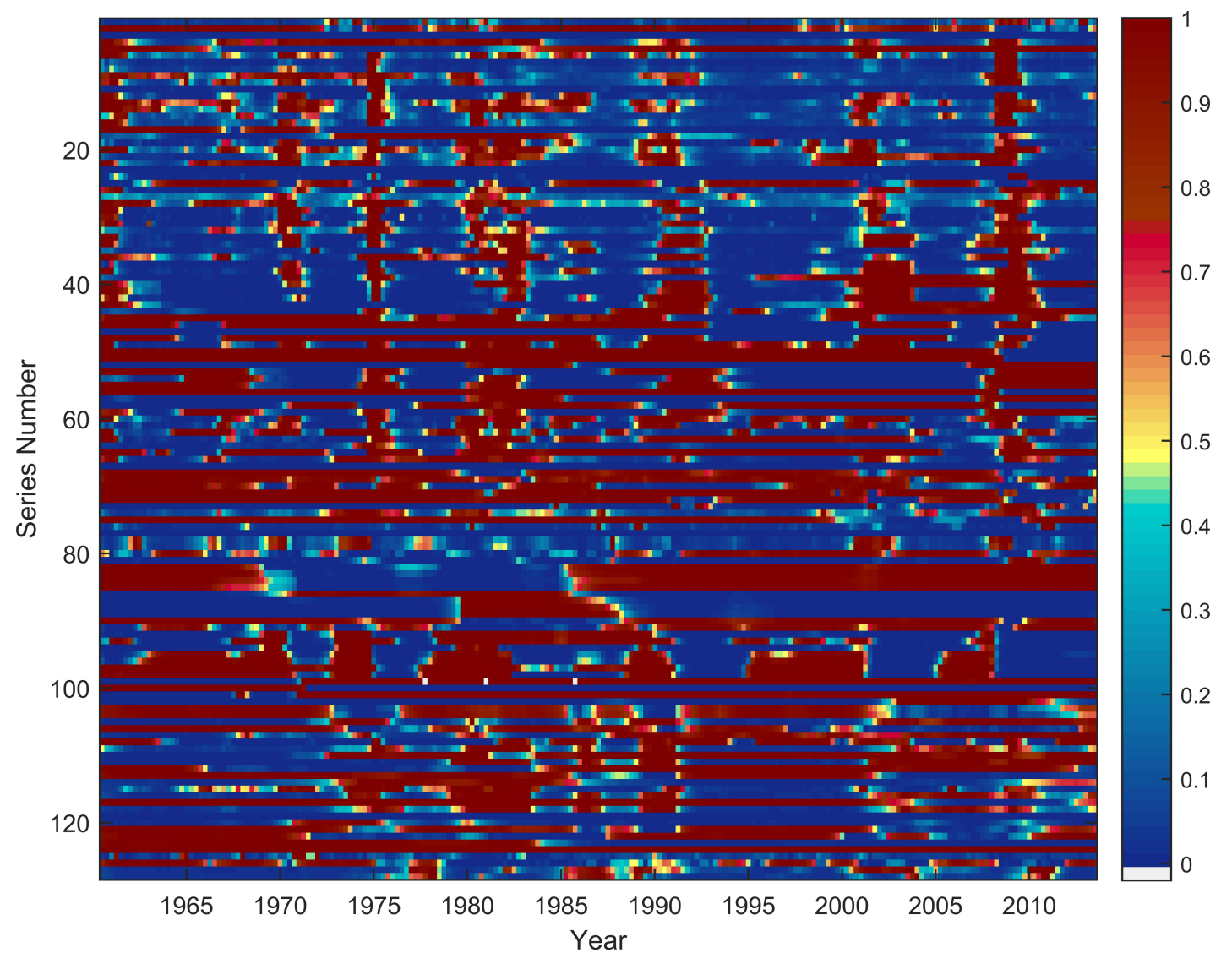

Note: Dark red indicates higher value for the probability of being in the first regime, which is normalized to correspond to the lowest intercept of the two regimes. Factor loadings are obtained from the MS-3PRF approach with GDP growth as a target proxy. 


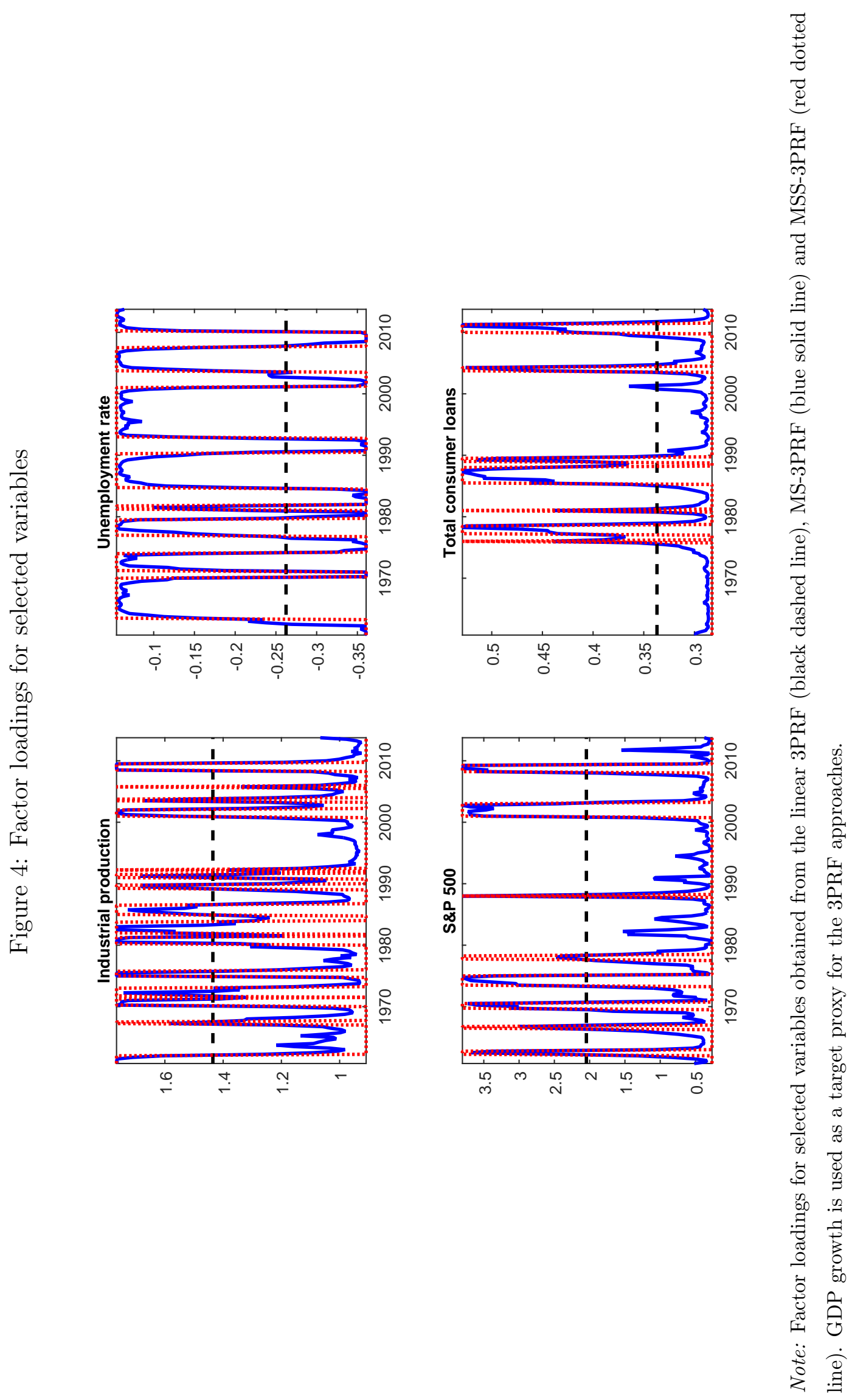


Table 1: Simulation results with different degrees of instabilities in the loadings

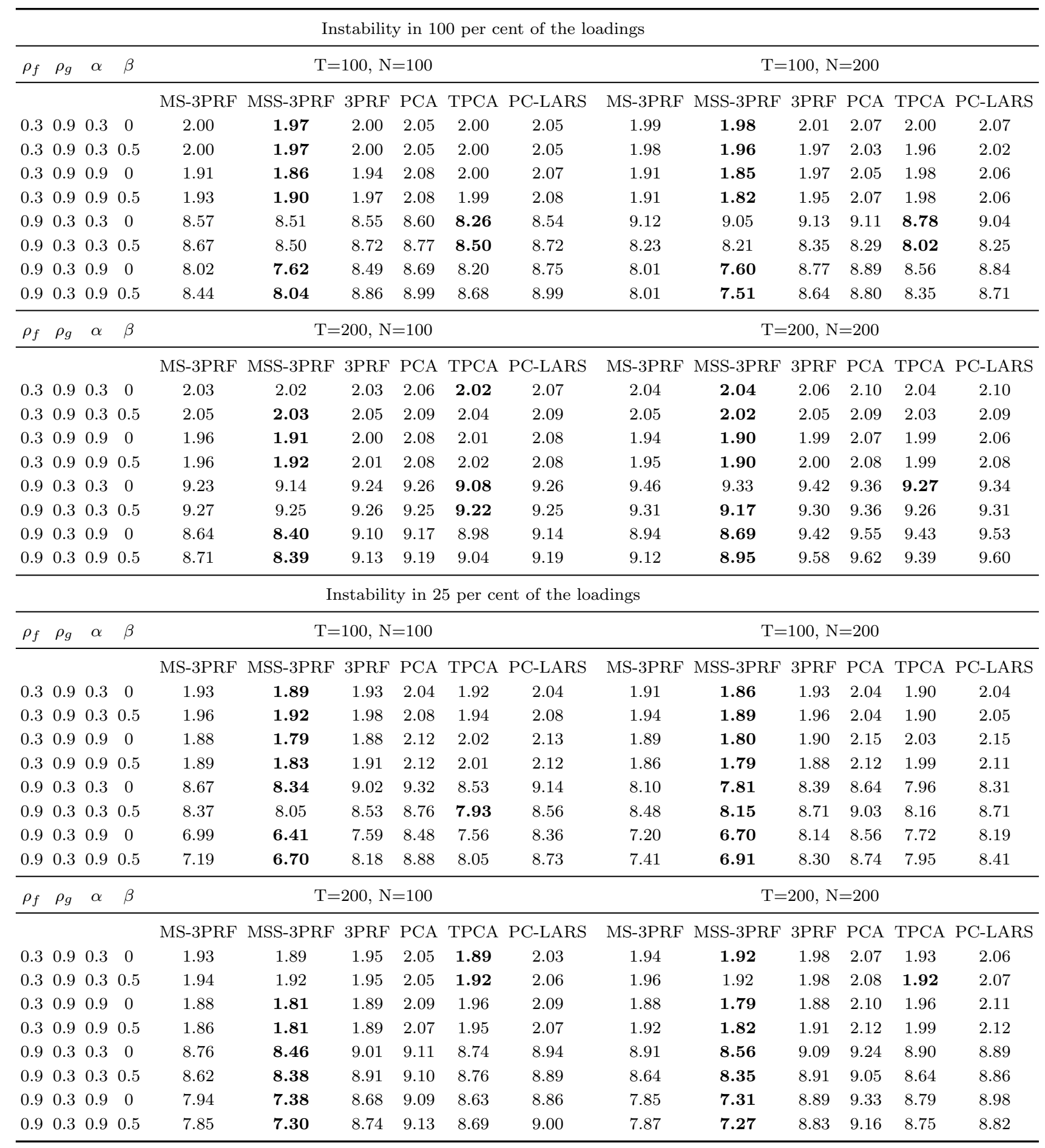

Note: The table reports the median MSFE based on 500 replications. Serial correlation in the factors is governed by $\rho_{f}$ and $\rho_{g}$, while $\alpha$ and $\beta$ govern serial and cross-sectional correlation in the predictors' residuals, respectively. Entries in bold represent the lowest median MSFE for each specification. See text for additional details. 
Table 2: Simulation results with different relationships of loadings instabilities

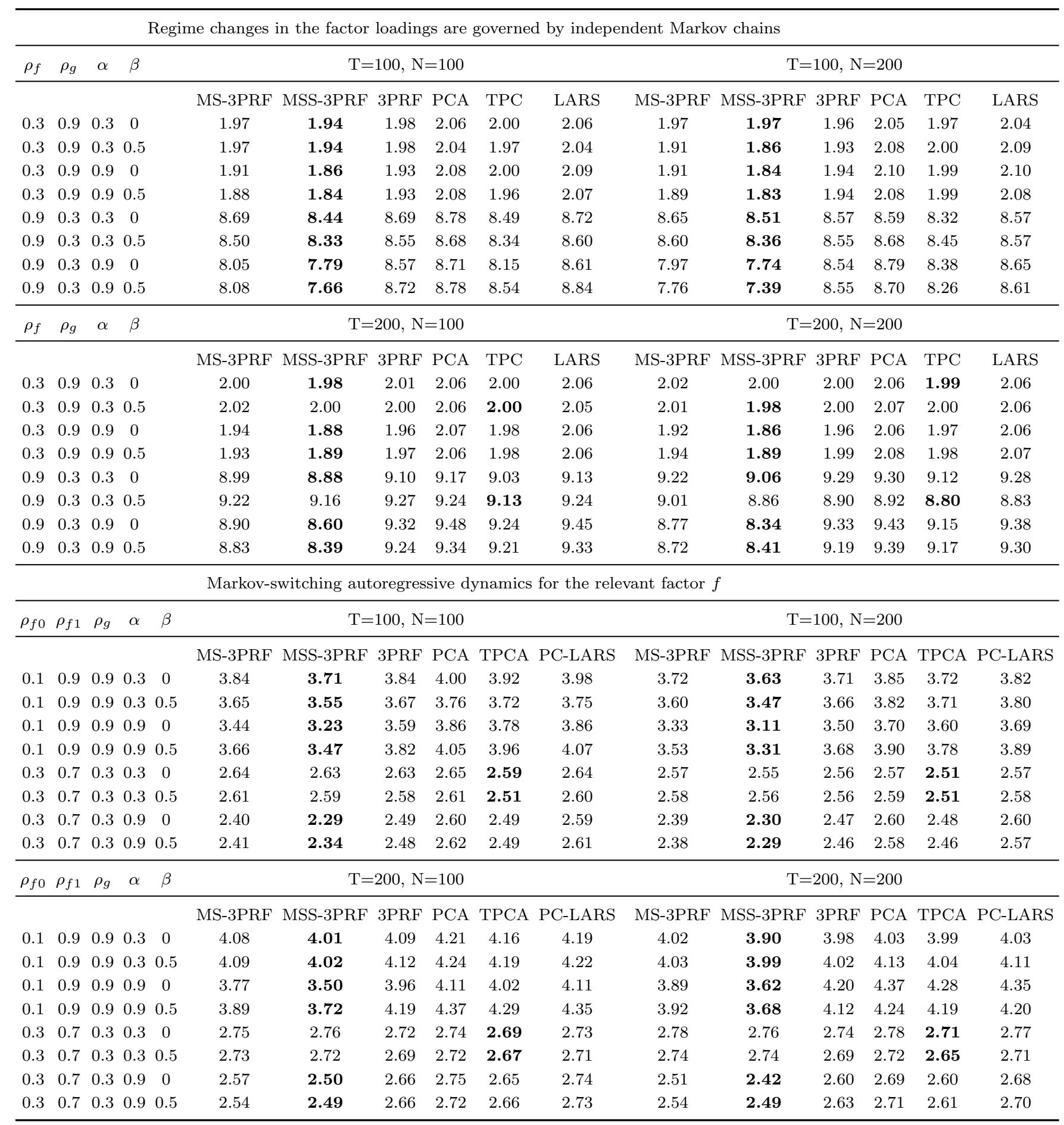

Note: The table reports the median MSFE based on 500 replications. Serial correlation in the relevant factor during each of the two regimes is governed by $\rho_{f 0}$ and $\rho_{f 1}$, and serial correlation in the irrelevant factors is governed by $\rho_{g}$, while $\alpha$ and $\beta$ govern serial and cross-sectional correlations in the predictors' residuals. Entries in bold represent the lowest median MSFE for each specification. See text for additional details. 
Table 3: Out-of-sample exchange rate forecasting - selected currencies (RMSFE)

\begin{tabular}{|c|c|c|c|c|c|c|}
\hline Forecast horizon & 1 & 2 & 3 & 6 & 9 & 12 \\
\hline \multicolumn{7}{|c|}{$\mathrm{CAD} / \mathrm{USD}$} \\
\hline PCA & $0.871 *$ & 0.951 & 0.980 & 1.001 & 1.094 & 1.175 \\
\hline TPCA & $0.872^{*}$ & 0.955 & 0.986 & 0.996 & 1.088 & 1.182 \\
\hline PC-LARS & 0.872 & 0.949 & 0.986 & 0.998 & 1.095 & 1.157 \\
\hline Linear 3PRF & $0.910^{*}$ & 0.959 & 0.997 & 1.001 & 1.043 & 1.057 \\
\hline MS-3PRF (first pass) & $0.915^{*}$ & 0.975 & 0.994 & 1.006 & 1.018 & 1.045 \\
\hline MS-3PRF (first and third passes) & 0.994 & 1.080 & 0.964 & 0.938 & 0.895 & 0.949 \\
\hline MSS-3PRF (first pass) & $0.916^{*}$ & 0.953 & 0.981 & 0.987 & 1.019 & 1.047 \\
\hline MSS-3PRF (first and third passes) & 1.150 & 1.204 & 0.995 & 0.972 & 1.148 & 1.072 \\
\hline \multicolumn{7}{|c|}{ EUR/USD } \\
\hline PCA & 0.988 & 1.033 & 1.000 & 1.034 & 1.085 & 1.138 \\
\hline TPCA & 0.995 & 1.049 & 1.010 & 1.061 & 1.109 & 1.155 \\
\hline PC-LARS & 0.961 & 1.028 & 1.010 & 1.072 & 1.141 & 1.207 \\
\hline Linear 3PRF & 1.014 & 1.010 & 1.016 & 1.049 & 1.081 & 1.148 \\
\hline MS-3PRF (first pass) & 0.986 & 1.028 & 1.011 & 1.022 & 1.071 & 1.127 \\
\hline MS-3PRF (first and third passes) & 1.078 & 1.037 & 1.117 & 1.170 & 1.126 & 1.270 \\
\hline MSS-3PRF (first pass) & 0.996 & 1.049 & 1.049 & 1.088 & 1.111 & 1.174 \\
\hline MSS-3PRF (first and third passes) & 1.234 & 1.108 & 1.306 & 1.329 & 1.463 & 2.043 \\
\hline \multicolumn{7}{|c|}{ JPY/USD } \\
\hline $\mathrm{PCA}$ & 1.091 & 1.093 & 1.108 & 1.070 & 1.101 & 1.145 \\
\hline TPCA & 1.081 & 1.111 & 1.098 & 1.066 & 1.085 & 1.121 \\
\hline PC-LARS & 1.066 & 1.066 & 1.078 & 1.078 & 1.074 & 1.117 \\
\hline Linear $3 \mathrm{PRF}$ & 1.019 & 1.046 & 1.055 & 1.031 & 1.068 & 1.105 \\
\hline MS-3PRF (first pass) & 1.019 & 1.035 & 1.038 & 1.055 & 1.075 & 1.094 \\
\hline MS-3PRF (first and third passes) & 1.035 & 0.984 & 1.025 & 1.126 & 0.879 & $0.877^{*}$ \\
\hline MSS-3PRF (first pass) & 1.032 & 1.023 & 1.024 & 1.045 & 1.082 & 1.072 \\
\hline MSS-3PRF (first and third passes) & 1.119 & 1.075 & 1.187 & 1.236 & $0.695 * *$ & 0.872 \\
\hline \multicolumn{7}{|c|}{ GBP/USD } \\
\hline PCA & $0.803^{*}$ & 0.977 & 1.043 & 1.045 & 1.064 & 1.091 \\
\hline TPCA & $0.796^{*}$ & 0.965 & 1.023 & 1.043 & 1.064 & 1.091 \\
\hline PC-LARS & $0.784^{*}$ & 0.953 & 1.029 & 1.035 & 1.073 & 1.114 \\
\hline Linear $3 \mathrm{PRF}$ & $0.875^{*}$ & 0.934 & 0.989 & 1.029 & 1.059 & 1.094 \\
\hline MS-3PRF (first pass) & 0.893 & 0.980 & 1.014 & 1.034 & 1.084 & 1.110 \\
\hline MS-3PRF (first and third passes) & 1.679 & 1.201 & 1.049 & 1.082 & 1.131 & 1.079 \\
\hline MSS-3PRF (first pass) & 0.889 & 0.982 & 1.013 & 1.039 & 1.078 & 1.113 \\
\hline MSS-3PRF (first and third passes) & 1.337 & 1.382 & 1.351 & 1.422 & 1.408 & 1.252 \\
\hline
\end{tabular}

Note: This table shows the relative mean square forecast error (RMSFE) for selected currency pairs (CAD/USD, EUR/USD, JPY/USD and GBP/USD) using PCA, TPCA, PC-LARS, linear 3PRF, MS3PRF (first pass), MS-3PRF (first and third passes), MSS-3PRF (first pass) and MSS-3PRF (first and third passes) as forecasting approaches. An entry smaller than 1 indicates that a given approach outperforms the random-walk model. Entries in bold indicate the best-performing approach for a specific horizon. Statistically significant reductions in the MSFE relative to the random walk according to the DieboldMariano test are indicated by asterisks (* denotes significance at the 10 per cent level, and ** denotes significance at the 5 per cent level). 
Table 4: Out-of-sample exchange rate forecasting - selected currencies (success ratios)

\begin{tabular}{|c|c|c|c|c|c|c|}
\hline Forecast horizon & 1 & 2 & 3 & 6 & 9 & 12 \\
\hline \multicolumn{7}{|c|}{$\mathrm{CAD} / \mathrm{USD}$} \\
\hline PCA & $0.637^{* *}$ & $0.602^{* *}$ & $0.575^{*}$ & 0.522 & 0.504 & 0.407 \\
\hline TPCA & $0.637^{* *}$ & $0.620^{* *}$ & $0.593^{* *}$ & 0.522 & 0.487 & 0.407 \\
\hline PC-LARS & $0.611^{* *}$ & $0.602^{* *}$ & 0.513 & 0.487 & 0.469 & 0.407 \\
\hline Linear $3 \mathrm{PRF}$ & $0.611^{* *}$ & $0.620 * *$ & $0.558^{* *}$ & 0.513 & 0.522 & 0.478 \\
\hline MS-3PRF (first pass) & $0.620^{* *}$ & $0.575^{* *}$ & 0.540 & 0.487 & 0.522 & 0.460 \\
\hline MS-3PRF (first and third passes) & 0.558 & $0.566^{* *}$ & $0.690 * *$ & $0.655 * *$ & $0.646^{* *}$ & $0.673^{* *}$ \\
\hline MSS-3PRF (first pass) & $0.646^{* *}$ & $0.611^{* *}$ & 0.558 & 0.504 & 0.522 & 0.460 \\
\hline MSS-3PRF (first and third passes) & 0.504 & 0.522 & $0.620^{* *}$ & 0.575 & $0.673^{* *}$ & $0.681 * *$ \\
\hline \multicolumn{7}{|c|}{ EUR/USD } \\
\hline $\mathrm{PCA}$ & 0.549 & 0.487 & 0.540 & 0.504 & 0.416 & 0.372 \\
\hline TPCA & 0.558 & 0.487 & 0.566 & 0.487 & 0.443 & 0.354 \\
\hline PC-LARS & $0.566^{*}$ & 0.531 & 0.549 & 0.540 & 0.460 & 0.389 \\
\hline Linear 3PRF & 0.531 & 0.496 & 0.540 & 0.496 & 0.434 & 0.398 \\
\hline MS-3PRF (first pass) & 0.549 & 0.513 & 0.584 & 0.540 & 0.487 & 0.460 \\
\hline MS-3PRF (first and third passes) & 0.540 & 0.496 & 0.522 & 0.451 & 0.425 & 0.398 \\
\hline MSS-3PRF (first pass) & 0.549 & 0.496 & 0.540 & 0.443 & 0.460 & 0.416 \\
\hline MSS-3PRF (first and third passes) & 0.496 & 0.575 & 0.540 & 0.487 & 0.425 & 0.345 \\
\hline \multicolumn{7}{|c|}{ JPY/USD } \\
\hline PCA & 0.460 & 0.496 & 0.469 & 0.496 & 0.504 & 0.425 \\
\hline TPCA & 0.460 & 0.434 & 0.451 & 0.504 & 0.478 & 0.425 \\
\hline PC-LARS & 0.531 & 0.504 & 0.496 & 0.487 & 0.469 & 0.425 \\
\hline Linear $3 \mathrm{PRF}$ & $0.575^{* *}$ & $0.549^{*}$ & 0.531 & 0.522 & 0.460 & 0.504 \\
\hline MS-3PRF (first pass) & 0.496 & 0.487 & 0.443 & 0.443 & 0.487 & 0.460 \\
\hline MS-3PRF (first and third passes) & $0.593^{* *}$ & $0.575^{*}$ & 0.487 & 0.522 & $0.726^{* *}$ & 0.584 \\
\hline MSS-3PRF (first pass) & 0.513 & 0.513 & 0.531 & 0.531 & 0.407 & 0.469 \\
\hline MSS-3PRF (first and third passes) & 0.504 & $0.566^{* *}$ & 0.496 & 0.566 & $0.797^{* *}$ & $0.611 *$ \\
\hline \multicolumn{7}{|c|}{ GBP/USD } \\
\hline $\mathrm{PCA}$ & $0.593^{* *}$ & 0.540 & 0.513 & 0.434 & 0.522 & 0.575 \\
\hline TPCA & $0.593^{* *}$ & 0.540 & 0.504 & 0.469 & $0.558 * *$ & $0.584^{* *}$ \\
\hline PC-LARS & $0.611 * *$ & $0.593^{* *}$ & 0.531 & 0.531 & 0.425 & 0.496 \\
\hline Linear 3PRF & $0.584^{* *}$ & $0.593 * *$ & $0.602^{* *}$ & 0.513 & 0.504 & 0.478 \\
\hline MS-3PRF (first pass) & 0.549 & $0.566^{*}$ & 0.549 & 0.504 & 0.504 & 0.451 \\
\hline MS-3PRF (first and third passes) & 0.460 & 0.540 & $0.593^{* *}$ & 0.504 & 0.425 & 0.522 \\
\hline MSS-3PRF (first pass) & 0.522 & 0.522 & 0.558 & 0.504 & 0.531 & 0.558 \\
\hline MSS-3PRF (first and third passes) & 0.460 & 0.522 & 0.531 & 0.496 & 0.416 & 0.504 \\
\hline
\end{tabular}

Note: This table shows the success ratios for selected currency pairs (CAD/USD, EUR/USD, JPY/USD and GBP/USD) using PCA, TPCA, PC-LARS, linear 3PRF, MS-3PRF (first pass), MS-3PRF (first and third passes), MSS-3PRF (first pass) and MSS-3PRF (first and third passes) as forecasting approaches. Under the null hypothesis of no directional accuracy, one would expect a success ratio of 0.5. Higher ratios indicate an improvement over the no-change forecast. Entries in bold indicate the best-performing approach for a specific horizon. Statistically significant improvements in directional accuracy according to the Pesaran-Timmermann test are indicated by asterisks (* denotes significance at the 10 per cent level, and ${ }^{* *}$ denotes significance at the 5 per cent level). 
Table 5: Out-of-sample macroeconomic forecasting

\begin{tabular}{|c|c|c|c|c|c|c|c|c|}
\hline Forecast horizon & 1 & 2 & 3 & 4 & 5 & 6 & 7 & 8 \\
\hline \multicolumn{9}{|c|}{$G D P$} \\
\hline MS-3PRF (first pass) & 1.151 & $0.967^{*}$ & $0.975^{*}$ & 0.973 & $0.936 * *$ & 0.976 & 1.013 & 0.979 \\
\hline MS-3PRF (first and third passes) & 1.095 & 1.029 & 1.306 & 1.196 & 1.026 & 1.147 & 1.169 & 1.104 \\
\hline MSS-3PRF (first pass) & 1.150 & $0.961 * *$ & $0.975^{*}$ & $0.943^{* *}$ & $0.943^{* *}$ & 0.973 & 1.020 & 0.959 \\
\hline MSS-3PRF (first and third passes) & 0.962 & 1.114 & 1.172 & 1.246 & 1.226 & 1.172 & 1.118 & 1.109 \\
\hline Linear 3PRF & 1.077 & $0.971^{* *}$ & 1.009 & 0.983 & $0.943^{* *}$ & $0.945 * *$ & $0.978 * *$ & $0.916 * *$ \\
\hline TPCA & $0.966^{* *}$ & $0.986^{* *}$ & $0.990 * *$ & 0.996 & 0.997 & 1.006 & 1.003 & 1.004 \\
\hline PC-LARS & 1.024 & $0.963^{*}$ & $0.950^{* *}$ & $0.935 * *$ & $0.944^{* *}$ & 0.962 & 1.003 & $0.950^{*}$ \\
\hline \multicolumn{9}{|c|}{ Consumption } \\
\hline MS-3PRF (first pass) & 1.054 & 0.963 & 1.001 & 0.933 & 0.950 & 0.956 & 0.955 & 0.956 \\
\hline MS-3PRF (first and third passes) & 1.073 & 1.029 & 1.368 & 1.099 & 1.164 & 1.058 & 1.109 & 1.168 \\
\hline MSS-3PRF (first pass) & 1.035 & 0.958 & 0.987 & 0.920 & 0.956 & $0.944^{*}$ & 0.952 & 0.953 \\
\hline MSS-3PRF (first and third passes) & 1.055 & 1.149 & 1.256 & 1.421 & 1.309 & 1.467 & 1.300 & 1.311 \\
\hline Linear 3PRF & 0.970 & $0.959 * *$ & $0.969 * *$ & $0.949^{* *}$ & $0.954^{* *}$ & $0.926 * *$ & $0.939 * *$ & $0.945 * *$ \\
\hline TPCA & $0.939 * *$ & $0.961^{* *}$ & $0.981^{* *}$ & $0.986^{*}$ & 0.997 & 1.008 & 1.020 & 1.009 \\
\hline PC-LARS & 1.028 & 1.011 & $0.972^{* *}$ & 1.016 & 0.982 & 0.990 & 0.961 & 0.985 \\
\hline \multicolumn{9}{|c|}{ Investment } \\
\hline MS-3PRF (first pass) & 0.933 & 0.996 & 1.039 & 1.039 & 1.000 & 0.995 & 1.013 & 1.010 \\
\hline MS-3PRF (first and third passes) & 1.181 & 1.106 & 1.186 & 1.397 & 1.207 & 1.140 & 1.104 & 1.065 \\
\hline MSS-3PRF (first pass) & 0.963 & 0.998 & 1.047 & 1.056 & 1.001 & 1.006 & 1.015 & 1.015 \\
\hline MSS-3PRF (first and third passes) & 0.912 & 1.062 & 1.141 & 1.139 & 1.081 & 1.043 & 1.029 & 1.043 \\
\hline Linear 3PRF & 0.997 & 0.990 & 1.034 & 1.023 & 0.996 & 0.989 & 0.992 & 0.988 \\
\hline TPCA & 1.010 & 0.998 & 0.999 & 1.001 & 1.005 & 1.009 & 1.003 & 1.005 \\
\hline PC-LARS & 0.913 & 0.977 & 0.980 & 0.991 & 0.995 & 1.003 & 1.021 & 1.007 \\
\hline \multicolumn{9}{|c|}{ Export } \\
\hline MS-3PRF (first pass) & 1.175 & 0.984 & 1.009 & 0.963 & 1.020 & 1.004 & 1.007 & 0.982 \\
\hline MS-3PRF (first and third passes) & 1.003 & 1.031 & 1.108 & 1.082 & 1.019 & 1.084 & 1.003 & $0.954^{* *}$ \\
\hline MSS-3PRF (first pass) & 1.192 & 0.985 & 0.998 & 0.965 & 1.015 & 1.008 & 1.003 & 0.989 \\
\hline MSS-3PRF (first and third passes) & $0.927 * *$ & 1.072 & 1.094 & 1.037 & 1.036 & 1.057 & 1.064 & 1.045 \\
\hline Linear 3PRF & 1.003 & 0.996 & 0.991 & 0.983 & 1.003 & 0.983 & 0.996 & 0.999 \\
\hline TPCA & 1.061 & 1.029 & 1.021 & 1.006 & 1.008 & 1.002 & 0.999 & 0.998 \\
\hline PC-LARS & 1.117 & 1.027 & 1.018 & 1.000 & 1.010 & 1.003 & 1.006 & 1.003 \\
\hline
\end{tabular}

Note: This table reports the MSFE of a given approach relative to the MSFE of PCA for forecast horizons ranging from one quarter to eight quarters ahead. Linear 3PRF uses a single target proxy. MS-3PRF (first pass) and MSS-3PRF (first pass) are regime-switching 3PRFs based on a single target proxy and regime-switching parameters in the first pass only; MS-3PRF (first and third passes) and MSS-3PRF (first and third passes) are regime-switching 3PRFs based on a single target proxy and regime-switching parameters in the first and third passes. For these approaches, the target proxy is the variable to forecast. TPCA is PCA where hard thresholding was performed before extracting the first principal component to forecast. PC-LARS is PCA where soft thresholding was performed before extracting the first principal component to forecast. Boldface indicates the best-performing procedure for a specific horizon and variable. The first estimation sample extends from 1960Q3 to $1984 \mathrm{Q} 4$, and it is recursively expanded as we progress in the forecasting exercise. The full evaluation sample runs from 1985Q1 to 2015Q3. Statistical reductions in MSFE relative to PCA according to the Diebold and Mariano (1995) test are indicated by asterisks (* denotes significance at the 10 per cent level, and ${ }^{* *}$ denotes significance at the 5 per cent level). 
Table 6: Out-of-sample macroeconomic forecasting

\begin{tabular}{|c|c|c|c|c|c|c|c|c|}
\hline Forecast horizon & 1 & 2 & 3 & 4 & 5 & 6 & 7 & 8 \\
\hline \multicolumn{9}{|c|}{ Import } \\
\hline MS-3PRF (first pass) & 1.129 & 0.981 & 0.938 & 0.994 & $0.921 *$ & 1.011 & 0.993 & 0.948 \\
\hline MS-3PRF (first and third passes) & 0.992 & 0.947 & 0.925 & 0.987 & 0.928 & 0.967 & 1.009 & 0.981 \\
\hline MSS-3PRF (first pass) & 1.187 & 0.983 & 0.935 & 0.997 & $0.916^{*}$ & 1.007 & 1.026 & 0.953 \\
\hline MSS-3PRF (first and third passes) & 1.035 & 0.961 & 0.963 & 1.051 & 0.973 & 1.026 & 1.062 & 1.027 \\
\hline Linear 3PRF & 1.028 & 0.994 & 0.976 & $0.978^{*}$ & $0.943^{*}$ & $0.936^{* *}$ & $0.962^{*}$ & $0.940 *$ \\
\hline TPCA & 1.012 & $0.995^{* *}$ & 0.997 & 1.004 & 1.012 & 1.011 & 1.010 & 1.018 \\
\hline PC-LARS & 1.058 & 0.991 & 1.004 & 1.000 & 0.977 & 0.977 & $0.962 * *$ & 0.972 \\
\hline \multicolumn{9}{|c|}{ Hours } \\
\hline MS-3PRF (first pass) & 1.057 & 1.037 & 1.013 & 0.995 & 0.992 & 1.006 & 0.972 & 0.982 \\
\hline MS-3PRF (first and third passes) & 1.037 & 1.253 & 1.565 & 1.500 & 1.417 & 1.159 & 1.327 & 1.217 \\
\hline MSS-3PRF (first pass) & 1.088 & 1.032 & 1.017 & 0.995 & 0.997 & 1.011 & 0.984 & 0.990 \\
\hline MSS-3PRF (first and third passes) & 1.069 & 1.001 & 1.017 & 1.109 & 1.319 & 1.401 & 1.515 & 1.491 \\
\hline Linear 3PRF & 1.023 & 1.026 & 1.046 & 1.023 & 1.009 & 1.009 & 1.003 & 0.992 \\
\hline TPCA & 1.010 & 1.001 & 1.003 & 1.005 & 1.009 & 1.003 & 1.005 & 1.008 \\
\hline PC-LARS & $0.864^{* *}$ & $0.980 *$ & 0.993 & 1.006 & 1.010 & 1.004 & 1.017 & 1.030 \\
\hline \multicolumn{9}{|c|}{ GDP inflation } \\
\hline MS-3PRF (first pass) & 1.018 & 1.094 & 1.215 & 1.242 & 1.289 & 1.296 & 1.312 & 1.320 \\
\hline MS-3PRF (first and third passes) & 1.003 & 1.099 & 1.725 & 1.611 & 1.528 & 1.393 & 1.407 & 1.459 \\
\hline MSS-3PRF (first pass) & 1.018 & 1.096 & 1.181 & 1.228 & 1.263 & 1.276 & 1.288 & 1.315 \\
\hline MSS-3PRF (first and third passes) & 0.965 & 0.787 & 0.798 & 0.784 & $0.710^{*}$ & $0.616 * *$ & $0.588 * *$ & $0.570 * *$ \\
\hline Linear $3 \mathrm{PRF}$ & 1.109 & 1.263 & 1.320 & 1.093 & 1.068 & 1.038 & 1.038 & 1.048 \\
\hline TPCA & 1.010 & 1.026 & 1.037 & 1.056 & 1.050 & 1.059 & 1.031 & 1.044 \\
\hline PC-LARS & 0.996 & 1.031 & 1.140 & 1.294 & 1.250 & 1.221 & 1.132 & 1.085 \\
\hline \multicolumn{9}{|c|}{$P C E$ inflation } \\
\hline MS-3PRF (first pass) & 1.033 & 1.029 & 1.069 & 1.059 & 1.051 & 1.102 & 1.124 & 1.104 \\
\hline MS-3PRF (first and third passes) & 1.004 & 1.065 & 1.547 & 1.867 & 2.076 & 2.353 & 2.469 & 2.346 \\
\hline MSS-3PRF (first pass) & 1.020 & 1.020 & 1.056 & 1.050 & 1.028 & 1.085 & 1.093 & 1.050 \\
\hline MSS-3PRF (first and third passes) & 0.957 & 0.863 & 0.938 & $0.834^{*}$ & $0.801 * *$ & $0.835^{*}$ & $0.778^{* *}$ & $0.707 * *$ \\
\hline Linear 3PRF & 1.038 & 1.045 & 1.074 & 1.049 & 1.076 & 1.100 & 1.082 & 1.114 \\
\hline TPCA & 1.031 & 1.023 & 1.025 & 1.023 & 1.029 & 1.028 & 1.049 & 1.043 \\
\hline PC-LARS & 1.069 & 1.130 & 1.169 & 1.168 & 1.132 & 1.100 & 1.077 & 1.046 \\
\hline
\end{tabular}

Note: This table reports the MSFE of a given approach relative to the MSFE of PCA for forecast horizons ranging from one quarter to eight quarters ahead. Linear 3PRF uses a single target proxy. MS-3PRF (first pass) and MSS-3PRF (first pass) are regime-switching 3PRFs based on a single target proxy and regime-switching parameters in the first pass only; MS-3PRF (first and third passes) and MSS-3PRF (first and third passes) are regime-switching 3PRFs based on a single target proxy and regime-switching parameters in the first and third passes. For these approaches, the target proxy is the variable to forecast. TPCA is PCA where hard thresholding was performed before extracting the first principal component to forecast. PC-LARS is PCA where soft thresholding was performed before extracting the first principal component to forecast. Boldface indicates the best-performing procedure for a specific horizon and variable. The first estimation sample extends from 1960Q3 to $1984 \mathrm{Q} 4$, and it is recursively expanded as we progress in the forecasting exercise. The full evaluation sample runs from 1985Q1 to 2015Q3. Statistical reductions in MSFE relative to PCA according to the Diebold and Mariano (1995) test are indicated by asterisks (* denotes significance at the 10 per cent level, and ${ }^{* *}$ denotes significance at the 5 per cent level). 